\title{
Convergence analysis for a conformal discretization of a model for precipitation and dissolution in porous media
}

Citation for published version (APA):

Kumar, K., Pop, I. S., \& Radu, F. A. (2012). Convergence analysis for a conformal discretization of a model for precipitation and dissolution in porous media. (CASA-report; Vol. 1208). Technische Universiteit Eindhoven.

Document status and date:

Published: 01/01/2012

\section{Document Version:}

Publisher's PDF, also known as Version of Record (includes final page, issue and volume numbers)

\section{Please check the document version of this publication:}

- A submitted manuscript is the version of the article upon submission and before peer-review. There can be important differences between the submitted version and the official published version of record. People interested in the research are advised to contact the author for the final version of the publication, or visit the $\mathrm{DOI}$ to the publisher's website.

- The final author version and the galley proof are versions of the publication after peer review.

- The final published version features the final layout of the paper including the volume, issue and page numbers.

Link to publication

\section{General rights}

Copyright and moral rights for the publications made accessible in the public portal are retained by the authors and/or other copyright owners and it is a condition of accessing publications that users recognise and abide by the legal requirements associated with these rights.

- Users may download and print one copy of any publication from the public portal for the purpose of private study or research.

- You may not further distribute the material or use it for any profit-making activity or commercial gain

- You may freely distribute the URL identifying the publication in the public portal.

If the publication is distributed under the terms of Article 25fa of the Dutch Copyright Act, indicated by the "Taverne" license above, please follow below link for the End User Agreement:

www.tue.nl/taverne

Take down policy

If you believe that this document breaches copyright please contact us at:

openaccess@tue.nl

providing details and we will investigate your claim. 


\section{EINDHOVEN UNIVERSITY OF TECHNOLOGY}

Department of Mathematics and Computer Science

\section{CASA-Report I2-08 \\ April 2012}

\section{Convergence analysis for a conformal discretization of a model for precipitation and dissolution in porous media}

by

K. Kumar, I.S. Pop, F.A. Radu

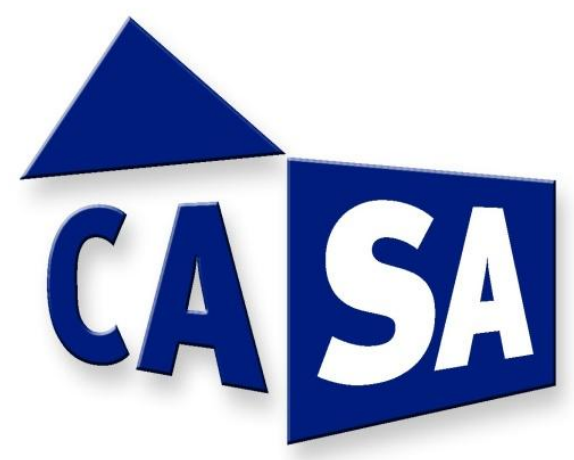

Centre for Analysis, Scientific computing and Applications

Department of Mathematics and Computer Science

Eindhoven University of Technology

P.O. Box 513

5600 MB Eindhoven, The Netherlands

ISSN: 0926-4507 



\title{
CONVERGENCE ANALYSIS FOR A CONFORMAL DISCRETIZATION OF A MODEL FOR PRECIPITATION AND DISSOLUTION IN POROUS MEDIA
}

\author{
K. KUMAR ${ }^{1}$, I. S. POP ${ }^{1}$, F. A. RADU ${ }^{2}$ \\ 1 CASA, TECHNISCHE UNIVERSITEIT EINDHOVEN, EINDHOVEN, THE NETHERLANDS \\ 2 INSTITUTE OF MATHEMATICS, JOHANNES BRUNS GT. 12, UNIVERSITY OF BERGEN, NORWAY
}

\begin{abstract}
In this paper we discuss the numerical analysis of an upscaled (core scale) model describing the transport, precipitation and dissolution of solutes in a porous medium. The particularity lies in the modeling of the reaction term, especially the dissolution term, which has a multivalued character. We consider the weak formulation for the upscaled equation and provide rigorous stability and convergence results for both the semi-discrete (time discretization) and the fully discrete scheme. In doing so, compactness arguments are employed.
\end{abstract}

Key words. Numerical analysis, reactive flows, weak formulation, implicit scheme, finite element discretization

AMS subject classifications. 35A35, 65L60, 65J20

1 Introduction In this paper we consider a model for the reactive flow in a porous medium, where the ions/solutes are being transported through the combined process of convection and diffusion. Such models are encountered in many real-life applications, like the spreading of chemical and the resulting ground water contamination (see [35] and references therein), biological applications such as tissue and bone formation, pharmaceutical applications [23], or the operation of solid state batteries. Of particular interest are the reactive processes, where precipitation and dissolution fronts develop as a result of reactions (see [24, 28, 30] and references therein). In a related context, [3] discusses the presence of stiff dissolution fronts involving the applications in the nuclear waste disposal.

Here we concentrate on a macroscale (upscaled) model, meaning that the model is defined at the Darcy scale. Therefore no distinction is made between the solid grains and the pore space, and the equations are defined everywhere in the domain of interest. The interesting aspect of the flow is the description of reactions taking place which have a particular structure. These reactions model the precipitation and dissolution processes taking place due to the interactions of ions (cations and anions). The reactions lead to the formation of crystal which are immobile species. Since, the model is considered at macroscale, both the crystals and the ions are defined everywhere. We take the model which was first proposed in [19] and then followed in a series of papers $[11,12,13]$. In [14], the corresponding pore scale model was presented. Further, the upscaled model is derived rigorously in a simplified situation of a $2 \mathrm{D}$ strip. For a similar situation, but now involving free boundaries at the pore scale, formal upscaling has been discussed in [20] and [25].

Our main goal here is to provide the convergence of a conforming FEM discretization for an upscaled model for dissolution and precipitation in porous media, involving a multi-valued dissolution rate. Before discussing the details, we briefly review some of the numerical work that is related to the present context. Conformal FEM schemes for reactive porous media flow models are discussed in [1,2], where nonLipschitz, but Hölder continuous rates are considered. Similarly, for Hölder continuous rates (including equilibrium and non-equilibrium cases) mixed FEM methods are analyzed rigorously in [31,32], whereas [33] provides error estimates for the coupled system describing unsaturated flow and reactive transport. In all these cases, the continuity of the reaction rates allows obtaining error estimates. Further, for continuously differentiable rates, the convergence of (adaptive) finite volume discretizations is studied in [18, 27]; see also [5] for the convergence of a finite volume discretization of a copper-leaching model. In a similar framework, discontinuous Galerkin methods are discussed in [34] and upwind mixed FEM are considered in $[8,9]$; combined finite volume-mixed hybrid finite elements are employed in $[16,17]$.

The primary motivation for the work here is to develop and analyse the appropriate numerical schemes to compute the solutions of macroscale equations. In the treatment here, we assume the flow to be given. Our primary focus is therefore to deal with the convection-diffusion-reaction equation where the non-linearities are in the reaction term. To avoid dealing with the inclusions as the dissolution rate is multi-valued, we use approximation schemes and consider both the semi-discrete and the fully discrete numerical schemes along 
these approximations (regularization). The resulting discrete equations are non-linear and the solutions depend on the regularization parameter. We prove their convergence to the time-continuous macroscale equations via a limiting procedure using compactness arguments. Whereas in the case of semi-discrete case, we use translation estimates to improve the convergence needed to deal with the non-linearities, in the fully-discrete case, we use the properties of Lagrange interpolation operator (see [7]) to achieve the required convergence. Of particular relevance to the work presented here is [10] where a semi-discrete numerical scheme for pore scale model is considered and the convergence is proved. Here, we consider both semi-discrete and fully discrete cases and for the latter we use finite element method to treat the spatial discretization. As a by-product of the convergence proof, we also obtain an alternative proof for existence of solutions for the macroscale equations. The present work should be considered as a first step towards an eventual plan to consider both the flow and the reactions coupled together (for example, Richards' equation coupled with precipitation-dissolution reaction models).

The paper is structured as follows. We begin with a brief description of the model in Section 2 where we also define the weak formulation of the model. We proceed to define the time-discrete formulation in Section 3 where the numerical scheme is analyzed and the convergence is proved. Next, in Section 4 , we consider the fully discrete formulation and treat the convergence issue. The numerical experiments are shown in Section 5 followed by the conclusions and discussions in Section 6.

2 The model We present here a brief description of the precipitation-dissolution model under consideration here. We refer to $[11,12,19]$ for the details of the model. It is relevant to mention that despite the simplification of the model under consideration, we retain the essence and thereby the interesting mathematical character of the model. Let $\Omega \subset \mathbb{R}^{2}$ be the domain occupied by the porous medium, and assume $\Omega$ be open, connected, bounded and polygonal with Lipschitz boundary $\Gamma$. Further, let $T>0$ be a fixed but arbitrarily chosen time, and define

$$
\Omega^{T}=(0, T] \times \Omega, \quad \text { and } \quad \Gamma^{T}=(0, T] \times \Gamma .
$$

While the reactions take place between the cations and anions, for the model considered here, we study only one mobile species (cation, though the choice is immaterial). This makes sense if the boundary and initial data are compatible (see [11], or [12]). Then, denoting by $v$ the concentration of the (immobile) precipitate, and by $u$ the cation concentration, the model reduces to

$$
\left\{\begin{aligned}
\partial_{t}(u+v)+\nabla \cdot(\mathbf{q} u-\nabla u) & =0, & & \text { in } \Omega^{T}, \\
u & =0, & & \text { on } \Gamma^{T}, \\
u & =u_{I}, & & \text { in } \Omega, \text { for } t=0,
\end{aligned}\right.
$$

for the ion transport, and

$$
\left\{\begin{aligned}
\partial_{t} v & =(r(u)-w), & & \text { on } \Omega^{T} \\
w & \in H(v), & & \text { on } \Omega^{T}, \\
v & =v_{I}, & & \text { on } \Omega, \text { for } t=0
\end{aligned}\right.
$$

for the precipitate. The rate of change in the precipitate concentration is the net process of precipitation and dissolution. Here $\mathbf{q}$ stands for the Darcy fluid velocity. We assume that $\mathbf{q}$ is a known, divergence free velocity

$$
\nabla \cdot \mathbf{q}=0 \text { in } \Omega
$$

For the ease of presentation we restrict to homogeneous Dirichlet boundary conditions. The initial data $u_{I}$ and $v_{I}$ are assumed non-negative and essentially bounded. Moreover, for simplicity we assume that both $u_{I}, v_{I} \in H_{0}^{1}(\Omega)$, the space of $H^{1}$ functions defined on $\Omega$ and having a vanishing trace on $\Gamma$.

All quantities and variables in the above are assumed dimensionless. The diffusion is assumed 1, the extension to a positive definite diffusion tensor being straightforward. Further, we assume that the Damköhler number is scaled to 1 , as well as an eventual factor in the time derivative of $v$ in $(2.2)_{1}$, appearing in the transition (homogenization) from the pore scale to the core scale. For the precipitation rate $r$ we assume 
(A1) $r(\cdot): \mathbb{R} \rightarrow[0, \infty)$ is locally Lipschitz continuous in $\mathbb{R}$.

(A2) There exists a unique $u_{*} \geq 0$, such that

$$
r(u)=\left\{\begin{array}{l}
0 \quad \text { for } u \leq u_{*}, \\
\text { strictly increasing for } u \geq u_{*} \text { with } r(\infty)=\infty .
\end{array}\right.
$$

The dissolution rate has a particular structure. It is assumed constant (1, by scaling) at some $(t, x) \in \Omega^{T}$ where the precipitate is present, i.e. if $v(t, x)>0$. In the absence of the precipitate, the overall rate (precipitate minus dissolution) is either zero if the solute present there is insufficient to produce a net precipitation gain, or positive in case the solute exceeds certain threshold value. In the presence of the precipitate, the dissolution strength is constant as it is a surface process. Further, the absence of net precipitation gain under insufficient amount of solutes being present, is related to the time-scale of observation. The derivation of the precipitation-dissolution is based on chemical kinetics and the ideas of solubility product for the crystals. For further discussions and derivation of this model, we refer to $[13,19]$. For the dissolution process, the rate law can be summarized as

$$
w \in H(v), \quad \text { where } H(v)=\left\{\begin{aligned}
0, & \text { if } v<0 \\
{[0,1], } & \text { if } v=0 \\
1, & \text { if } v>0 .
\end{aligned}\right.
$$

REMARK 2.1. Since the precipitation rate $r$ is monotonically increasing, under the setting above, a unique $u^{*}$ exists for which $r\left(u^{*}\right)=1$. Then $u^{*}$ can be interpreted as an equilibrium value: within an open set $A \subset \Omega^{T}$ where $u=u^{*}$, no precipitation or dissolution occurs, and the precipitation rate is balanced by the dissolution rate regardless of the presence or absence of crystals. Then, as follows from [14, 19, 26],

$$
w=1 \quad \text { a.e. in } \mathrm{A} \text {. }
$$

REMARK 2.2. The upscaled model under discussion, proposed originally in [19] (see also [11, 12, 13]), and can be obtained by homogenization techniques, starting from the pore scale counterpart in [14].

We emphasize on the particularity of the present model, which is in the description of the dissolution and precipitation processes, involving a multi-valued dissolution rate. Clearly, classical solutions do not exist, except for some particular cases. Therefore we resort to defining appropriate weak solutions. We treat here the conformal weak formulation which one formally obtains by multiplying the equations, e.g. (2.1), (2.2) by smooth test functions and using partial integration, if necessary. We give a formal definition in Section 2.2 .

2.1 Notations and assumptions We adopt the following standard notations from the functional analysis. By $(\cdot, \cdot)$ we mean $L^{2}(\Omega)$ inner product or the duality pairing between $H_{0}^{1}$ and $H^{-1}$; the $L^{2}\left(\Omega^{T}\right)$ inner product is denoted by $(\cdot, \cdot)_{\Omega^{T}}$. Further, $\|\cdot\|$ stands for the norms induced by $L^{2}$ inner product, $L^{2}(0, T ; X)$ denotes the usual Bochner spaces for a given Banach space $X$. For other norms, we explicitly state it. Furthermore, $C$ denotes the generic constant and the value of which might change from line to line and is independent of unknown variables or the discretization parameters. Let $L_{r}$ denote the Lipschitz constant of $r$ and $\|\mathbf{q}\|_{L^{\infty}(\Omega)} \leq M_{q}$ where $M_{q}$ is known.

We assume $\Omega \subset \mathbb{R}^{2}$ to be an open bounded and polygonal subset and define the following sets

$$
\begin{aligned}
\mathcal{U} & :=\left\{u \in L^{2}\left((0, T) ; H_{0}^{1}(\Omega)\right): \partial_{t} u \in L^{2}\left((0, T) ; H^{-1}(\Omega)\right)\right\} \\
\mathcal{V} & :=\left\{v \in H^{1}\left((0, T) ; L^{2}(\Omega)\right)\right\} \\
\mathcal{W} & :=\left\{w \in L^{\infty}\left(\Omega^{T}\right): 0 \leq w \leq 1\right\} .
\end{aligned}
$$

Since, $\Omega$ is polygonal, it has a regular decomposition into triangles and the errors due to nonpolygonal domains are avoided. Let $\mathcal{T}_{h}$ be a regular decomposition of $\Omega \subset \mathbb{R}^{2}$ into closed triangles; $h$ stands for the mesh-size. For the fully discrete situation, we will use the discrete subspace $\mathcal{U}_{h} \subset H_{0}^{1}(\Omega)$ defined as

$$
\mathcal{U}_{h}:=\left\{\theta \in C(\bar{\Omega}) \mid \theta \in \mathcal{P}_{1}(T), \quad T \in \mathcal{T}_{h}, \text { and } \theta=0 \text { on } \partial \Omega\right\},
$$


where $\mathcal{P}_{1}(T)$ is the space of first order polynomials in two variables, defined on a triangle $T$. In other words, $\mathcal{U}_{h}$ denotes the space of piecewise linear functions. Recall that $\mathcal{U}_{h} \subset H_{0}^{1}(\Omega)$ (see [6], p. 64). We also define the following projection:

$$
P_{h}: L^{2}(\Omega) \mapsto \mathcal{U}_{h},\left(P_{h} \theta-\theta, \psi_{h}\right)=0,
$$

for all $\psi_{h} \in \mathcal{U}_{h}$. Note that $P_{h}$ satisfies ([6], p. 138)

$$
\left\|P_{h} \theta-\theta\right\| \leq C h\|\nabla \theta\|,
$$

for all $\theta \in H_{0}^{1}(\Omega)$, for some $C>0$ not depending on $\theta$.

Moreover, let $\mathbf{q}_{h}$ be the discrete approximation of $\mathbf{q}$ and we assume $\nabla \cdot \mathbf{q}_{h}=0$ with

$$
\left\|\mathbf{q}-\mathbf{q}_{h}\right\| \searrow 0
$$

uniformly as $h \searrow 0$. Further, we assume that $\mathbf{q}_{h}$ also obeys the maximum principle so that $\left\|\mathbf{q}_{h}\right\|_{L^{\infty}(\Omega)} \leq$ $M_{q}$.

2.2 Weak formulation We start with defining the conformal weak formulation for (2.1)-(2.2).

DEFINITION 2.1. A triple $(u, v, w) \in \mathcal{U} \times \mathcal{V} \times \mathcal{W}$ is a weak solution of (2.1) and (2.2) if $\left(u_{\mid t=0}, v_{\mid t=0}\right)=$ $\left(u_{I}, v_{I}\right)$, and for all $(\phi, \theta) \in\left(L^{2}\left(0, T, H_{0}^{1}(\Omega)\right), L^{2}\left(0, T ; L^{2}(\Omega)\right)\right.$

$$
\begin{aligned}
\left(\partial_{t} u+\partial_{t} v, \phi\right)_{\Omega^{T}}+(\nabla u, \nabla \phi)_{\Omega^{T}}+(\boldsymbol{q} u, \nabla \phi)_{\Omega^{T}} & =0 \\
\left(\partial_{t} v, \theta\right)_{\Omega^{T}}-(r(u)-H(v), \theta)_{\Omega^{T}} & =0, \\
w & \in H(v), \text { a.e. } i n \Omega^{T} .
\end{aligned}
$$

The existence of a solution will be proved in Theorems 3.10 and 4.8 below. Alternatively, one can obtain the existence as an outcome of the rigorous homogenization procedure, starting from the model at the pore scale. The uniqueness follows by standard contraction arguments. This is summarized in the following

THEOREM 2.2. There exists a unique solution of (2.7) in the sense of Definition 2.1.

In what follows we propose numerical schemes for the above weak formulation and perform their analysis, namely proving the convergence of these discretized equations to the macroscale equations (2.7). First, we start with time-discrete formulation which we refer to as semi-discrete scheme. Consideration of this case provides a good understanding of the mathematical issues encountered in the convergence proofs and prepares for considering the fully-discrete case where we discretize in both space and time. The proofs in the latter case follow the same line of arguments as in the semi-discrete case and in some cases, the results can be directly borrowed. However, there are important differences and we comment on these as we proceed for the proofs.

As it has been remarked before, the study of numerical schemes and the convergence of these schemes to the macroscale equations provide us with an alternative proof for existence of solutions. We begin with the time-discrete formulation.

3 Semi-discrete scheme Before defining the time-discretization, let us note the presence of a multivalued rate in $(2.7)_{3}$, which impedes obtaining a priori estimates. Therefore we consider a regularized approximation of the original model (and pass later to the limit). We make sure that the estimates are independent of the regularization parameter, which is essential for passing to the limit. With $\delta>0$, define the regularized Heaviside function

$$
H_{\delta}(v)= \begin{cases}0, & \text { if } \quad v<0 \\ \frac{v}{\delta}, & \text { if } \quad 0 \leq v \leq \delta, \\ 1, & \text { if } \quad v>\delta .\end{cases}
$$

We start with defining the time-discrete scheme which we refer to $\mathbf{P}_{\delta}^{n}$ with subscript stressing the dependence of solution on the regularization parameter $\delta$. We then proceed to prove the convergence of the sequence of solutions of $\mathbf{P}_{\delta}^{n}$, passing to a subsequence, if necessary. Our steps for the proof of convergence follow the usual procedure. First, we obtain the a priori estimates which are independent of the discretization parameters and then define a time-continuous approximation for the solution. The estimates for these 
approximations then pave the way for using convergence results. In the wake of non-linearities involved, we need to improve the convergence, which we obtain by translation estimates in the semi-discrete situation.

Next, with $N \in \mathbb{N}, \tau=\frac{T}{N}$ and $t_{n}=n \tau, n=1, \ldots, N$, we consider a uniform time stepping that is implicit in $u$ and explicit in $v$. Starting with $u_{\delta}^{0}=u_{I}, v_{\delta}^{0}=v_{I}$, with $n \in\{1, \ldots, N\}$, the approximation $\left(u_{\delta}^{n}, v_{\delta}^{n}\right)$ of $\left(u\left(t_{n}\right), v\left(t_{n}\right)\right)$ solves

Problem $\mathbf{P}_{\delta}^{n}$ : Given $\left(u_{\delta}^{n-1}, v_{\delta}^{n-1}\right) \in\left(H_{0}^{1}(\Omega) \times L^{2}(\Omega)\right)$, find $\left(u_{\delta}^{n}, v_{\delta}^{n}\right) \in\left(H_{0}^{1}(\Omega) \times \in L^{2}(\Omega)\right)$ such that

$$
\begin{array}{r}
\left(\frac{u_{\delta}^{n}-u_{\delta}^{n-1}}{\tau}, \phi\right)+\left(\nabla u_{\delta}^{n}, \nabla \phi\right)-\left(\mathbf{q} u_{\delta}^{n}, \nabla \phi\right)+\left(\frac{v_{\delta}^{n}-v_{\delta}^{n-1}}{\tau}, \phi\right)=0 \\
\left(\frac{v_{\delta}^{n}-v_{\delta}^{n-1}}{\tau}, \theta\right)=\left(r\left(u_{\delta}^{n}\right)-H_{\delta}\left(v_{\delta}^{n-1}\right), \theta\right)
\end{array}
$$

for all $\phi \in H_{0}^{1}(\Omega), \theta \in L^{2}(\Omega)$. For completeness, we define

$$
w_{\delta}^{n}:=H_{\delta}\left(v_{\delta}^{n}\right) .
$$

For stability reasons, we choose $\delta=O\left(\tau^{\frac{1}{2}}\right)$ (see [10] for detailed arguments).

This is a system of elliptic equations for $u_{\delta}^{n}, v_{\delta}^{n}$ given $u_{\delta}^{n-1} \in H_{0}^{1}(\Omega), v_{\delta}^{n-1} \in L^{2}(\Omega)$. Note that the first equation is decoupled from the second equation since it can be written in the form

$$
\left(\frac{u_{\delta}^{n}-u_{\delta}^{n-1}}{\tau}, \phi\right)+\left(\nabla u_{\delta}^{n}, \nabla \phi\right)-\left(\mathbf{q} u_{\delta}^{n}, \nabla \phi\right)+\left(r\left(u_{\delta}^{n}\right)-H_{\delta}\left(v_{\delta}^{n-1}\right), \phi\right)=0 .
$$

With reaction term $r$ being Lipschitz and monotonic; standard monotonicity arguments can be used to show the existence and uniqueness of $u_{\delta}^{n}$ given $u_{\delta}^{n-1}$ [21]. After $u_{\delta}^{n}$ is computed, for the second equation, computing $v_{\delta}^{n}$ is straightforward as it is an explicit discretization in time. We summarize the above result:

Lemma 3.1. Problem $\boldsymbol{P}_{\delta}^{n}$ has a unique solution pair $\left(u_{\delta}^{n}, v_{\delta}^{n}\right)$.

For the continuous formulation, (2.7), $u, v$ are positive and essentially bounded. We proceed to establish that this property is also respected by the time-discrete formulation. With $M_{u}:=\max \left\{\left\|u_{I}\right\|_{L^{\infty}}, u^{*}\right\}$, $M_{v}:=\left\|v_{I}\right\|_{L^{\infty}}$, we note that $r\left(M_{u}\right) \geq 1$. Let us first prove the positivity of the solution pair $\left(u_{\delta}^{n}, v_{\delta}^{n}\right)$.

LEMMA 3.2. If $u_{\delta}^{n-1}, v_{\delta}^{n-1} \geq 0$ then $u_{\delta}^{n}, v_{\delta}^{n} \geq 0$.

Proof. We start with the estimate for $v_{\delta}^{n}$. In (3.2) $)_{2}$, for $\theta=\left[v_{\delta}^{n-1}\right]_{-}$, with $[\cdot]_{-}$denoting the non-positive part, we get

$$
\left\|\left[v_{\delta}^{n}\right]_{-}\right\|^{2}=\left(v_{\delta}^{n-1},\left[v_{\delta}^{n}\right]_{-}\right)+\tau\left(r\left(u_{\delta}^{n}\right)-H_{\delta}\left(v_{\delta}^{n-1}\right),\left[v_{\delta}^{n}\right]_{-}\right) .
$$

By assumption $v_{\delta}^{n-1} \geq 0, \delta=O\left(\tau^{\frac{1}{2}}\right)$ and $r\left(u_{\delta}^{n}\right) \geq 0$, we are left to consider

$$
v_{\delta}^{n-1}-\tau H_{\delta}\left(v_{\delta}^{n-1}\right) \geq v_{\delta}^{n-1}(1-\tau / \delta) \geq 0 .
$$

Hence $\left\|\left[v_{\delta}^{n}\right]_{-}\right\|^{2} \leq 0$ implying $v_{\delta}^{n} \geq 0$.

Next we prove that $u_{\delta}^{n}$ is nonnegative. For $\phi=\left[u_{\delta}^{n}\right]_{-}$in (3.2)

$$
\left\|\left[u_{\delta}^{n}\right]_{-}\right\|^{2}+\tau\left\|\nabla\left[u_{\delta}^{n}\right]_{-}\right\|^{2}-\tau\left(\mathbf{q} u_{\delta}^{n}, \nabla\left[u_{\delta}^{n}\right]_{-}\right)+\left(v_{\delta}^{n}-v_{\delta}^{n-1},\left[u_{\delta}^{n}\right]_{-}\right) \leq\left(u_{\delta}^{n-1},\left[u_{\delta}^{n}\right]_{-}\right) .
$$

The first two terms are nonnegative, whereas the third term vanishes:

$$
\left(\mathbf{q} u_{\delta}^{n}, \nabla\left[u_{\delta}^{n}\right]_{-}\right)=\frac{1}{2}\left(\mathbf{q}, \nabla\left[u_{\delta}^{n}\right]_{-}^{2}\right)=\frac{1}{2}\left(\nu \cdot \mathbf{q},\left[u_{\delta}^{n}\right]_{-}^{2}\right)_{\Gamma}-\frac{1}{2}\left(\nabla \cdot \mathbf{q},\left[u_{\delta}^{n}\right]_{-}^{2}\right)=0
$$

by the boundary conditions for $u_{\delta}^{n}$ on $\Gamma$ and $\nabla \cdot \mathbf{q}=0$ in $\Omega$. Further using (3.2) $)_{2}$ we have

$$
\left(v_{\delta}^{n}-v_{\delta}^{n-1},\left[u_{\delta}^{n}\right]_{-}\right)=\tau\left(r\left(u_{\delta}^{n}\right)-H_{\delta}\left(v_{\delta}^{n-1}\right),\left[u_{\delta}^{n}\right]_{-}\right)=-\tau\left(H_{\delta}\left(v_{\delta}^{n-1}\right),\left[u_{\delta}^{n}\right]_{-}\right) \geq 0
$$


where we have used the positivity of $r\left(u_{\delta}^{n-1}\right)$ and non-negativity of $H_{\delta}$. Now by assumption $u_{\delta}^{n-1} \geq 0$, we obtain $u_{\delta}^{n} \geq 0$.

The next lemma provides the pointwise bound for the concentration $u_{\delta}^{n}$ for any $n$. The following lemma and a simple induction argument give this bound.

LEMMA 3.3. If $u_{\delta}^{n-1} \leq M_{u}$ then $u_{\delta}^{n} \leq M_{u}$.

Proof. Test (3.2) $)_{1}$ with $\phi=\left[u_{\delta}^{n}-M_{u}\right]_{+}$(the non-negative part of $u_{\delta}^{n}-M_{u}$ ) to obtain

$$
\begin{array}{r}
\left\|\left[u_{\delta}^{n}-M_{u}\right]_{+}\right\|^{2}+\tau\left\|\nabla\left[u_{\delta}^{n}-M_{u}\right]_{+}\right\|^{2}-\tau\left(\mathbf{q} u_{\delta}^{n}, \nabla\left[u_{\delta}^{n}-M_{u}\right]_{+}\right) \\
+\left(v_{\delta}^{n}-v_{\delta}^{n-1},\left[u_{\delta}^{n}-M_{u}\right]_{+}\right) \leq\left(u_{\delta}^{n-1}-M_{u},\left[u_{\delta}^{n}-M_{u}\right]_{+}\right) .
\end{array}
$$

Again the third term vanishes using the arguments in the previous proof. Also

$$
\left(u_{\delta}^{n-1}-M_{u},\left[u_{\delta}^{n}-M_{u}\right]_{+}\right) \leq 0 \text { as } u_{\delta}^{n-1}-M_{u} \leq 0 .
$$

Furthermore,

$$
\left(v_{\delta}^{n}-v_{\delta}^{n-1},\left[u_{\delta}^{n}-M_{u}\right]_{+}\right)=\left(r\left(u_{\delta}^{n}\right)-H_{\delta}\left(v_{\delta}^{n-1}\right),\left[u_{\delta}^{n}-M_{u}\right]_{+}\right) \geq 0 \text { since } r\left(u_{\delta}^{n}\right)-H_{\delta}\left(v_{\delta}^{n-1}\right) \geq 0
$$

for $u_{\delta}^{n} \leq M_{u}$.

For the concentration of the crystal $v_{\delta}^{n}$, the situation is a bit different but expected as is evident from its ODE nature. Indeed, we have the following lemma:

LEMMA 3.4. Let $C=\frac{L_{r} M_{u}}{M_{v}}$ and assume that $v_{\delta}^{n-1} \leq M_{v} e^{C(n-1) \tau}$, then $v_{\delta}^{n} \leq M_{v} e^{C n \tau}$.

Proof. We use $\theta:=\left[v_{\delta}^{n}-M_{v} e^{C n \tau}\right]_{+}$in $(3.2)_{2}$ to obtain

$$
\begin{aligned}
\left\|\left[v_{\delta}^{n}-M_{v} e^{C n \tau}\right]_{+}\right\|^{2} & =\left(v_{\delta}^{n-1}-M_{v} e^{C(n-1) \tau},\left[v_{\delta}^{n}-M_{v} e^{C n \tau}\right]_{+}\right) \\
& +M_{v}\left(e^{C(n-1) \tau}-e^{C n \tau},\left[v_{\delta}^{n}-M_{v} e^{C n \tau}\right]_{+}\right) \\
& \tau\left(r\left(u_{\delta}^{n}\right)-H_{\delta}\left(v_{\delta}^{n-1}\right),\left[v_{\delta}^{n}-M_{v} e^{C n \tau}\right]_{+}\right) .
\end{aligned}
$$

Since, $r\left(u_{\delta}^{n}\right)$ is Lipschitz and $H_{\delta}(\cdot)$ is positive, we obtain

$$
\tau\left(r\left(u_{\delta}^{n}\right)-H_{\delta}\left(v_{\delta}^{n-1}\right),\left[v_{\delta}^{n}-M_{v} e^{C n \tau}\right]_{+}\right) \leq \tau\left(C M_{v},\left[v_{\delta}^{n}-M_{v} e^{C n \tau}\right]_{+}\right),
$$

and

$$
M_{v}\left(e^{C(n-1) \tau}-e^{C n \tau},\left[v_{\delta}^{n}-M_{v} e^{C n \tau}\right]_{+}\right) \leq M_{v}\left(1-e^{C \tau},\left[v_{\delta}^{n}-M_{v} e^{C n \tau}\right]_{+}\right) .
$$

Here, we have used $e^{C(n-1) \tau} \geq 1$. Next,

$$
\begin{array}{r}
\tau\left(C M_{v},\left[v_{\delta}^{n}-M_{v} e^{C n \tau}\right]_{+}\right)+M_{v}\left(1-e^{C \tau},\left[v_{\delta}^{n}-M_{v} e^{C n \tau}\right]_{+}\right) \\
\leq M_{v}\left(C \tau+1-e^{C \tau},\left[v_{\delta}^{n}-M_{v} e^{C n \tau}\right]_{+}\right) \leq 0
\end{array}
$$

since $1+x-e^{x} \leq 0$ for $x \geq 0$.

By assumption on $v_{\delta}^{n-1},\left(v_{\delta}^{n-1}-M_{v} e^{C(n-1) \tau},\left[v_{\delta}^{n}-M_{v} e^{C n \tau}\right]_{+}\right) \leq 0$ and using above in (3.4), we conclude

$$
\left\|\left[v_{\delta}^{n}-M_{v} e^{C n \tau}\right]_{+}\right\|^{2} \leq 0
$$

leading to the assertion. $\square$

As $n \tau \leq T$, we conclude that the estimates shown above are independent of $\delta$ and $\tau$. 
3.1 The a priori estimates With the pointwise bounds for the Problem $\mathbf{P}_{\delta}^{n}$ already established, we proceed to obtain energy estimates. These estimates will be used later for compactness arguments. These are similar to estimates for parabolic equations but here restricted to discrete time steps. We have the following lemma:

LEMMA 3.5. The following estimates hold

$$
\begin{array}{r}
\left\|v_{\delta}^{n}\right\| \leq\left\|v_{I}\right\|+C r\left(M_{u}\right), \\
\left\|v_{\delta}^{n}-v_{\delta}^{n-1}\right\| \leq C \tau, \\
\tau \sum_{n=1}^{N}\left\|\nabla u_{\delta}^{n}\right\|^{2} \leq C, \\
\sum_{n=1}^{N}\left\|u_{\delta}^{n}-u_{\delta}^{n-1}\right\|^{2} \leq C \tau, \\
\sum_{n=1}^{N}\left\|\nabla\left(u_{\delta}^{n}-u_{\delta}^{n-1}\right)\right\|^{2} \leq C,
\end{array}
$$

where $C$ is independent of $\tau$ and $\delta$.

Proof. To prove (3.5) we choose the test function $\phi=v_{\delta}^{n}$ in (3.2) $)_{1}$ to obtain

$$
\left(v_{\delta}^{n}-v_{\delta}^{n-1}, v_{\delta}^{n}\right)=\tau\left(r\left(u_{\delta}^{n}\right)-H_{\delta}\left(v_{\delta}^{n-1}\right), v_{\delta}^{n}\right),
$$

which leads to using Cauchy-Schwarz inequality and $H_{\delta}, v_{\delta}^{n} \geq 0$

$$
\left\|v_{\delta}^{n}\right\|^{2} \leq\left\|v_{\delta}^{n}\right\|\left(\left\|v_{\delta}^{n-1}\right\|+C \tau r\left(M_{u}\right)\right) .
$$

The above equation can be re-written as

$$
\left\|v_{\delta}^{n}\right\|-\left\|v_{\delta}^{n-1}\right\| \leq C \tau r\left(M_{u}\right)
$$

which we sum over $n$ to arrive at the assertion (3.5).

To prove (3.6), we consider (3.2) 2 and use $\theta=v_{\delta}^{n}-v_{\delta}^{n-1}$ to obtain

$$
\begin{aligned}
\left\|v_{\delta}^{n}-v_{\delta}^{n-1}\right\|^{2} & =\tau\left(r\left(u_{\delta}^{n}\right)-H_{\delta}\left(v_{\delta}^{n}\right), v_{\delta}^{n}-v_{\delta}^{n-1}\right) \\
& \leq \tau^{2} \frac{1}{2}\left(r\left(M_{u}\right)\right)^{2}+\frac{1}{2} \tau^{2}+\frac{1}{2}\left\|v_{\delta}^{n}-v_{\delta}^{n-1}\right\|^{2},
\end{aligned}
$$

leading to (3.6).

For (3.7), choosing $\phi=u_{\delta}^{n}$ in (3.2) 1 gives

$$
\left(u_{\delta}^{n}-u_{\delta}^{n-1}, u_{\delta}^{n}\right)+\tau\left\|\nabla u_{\delta}^{n}\right\|^{2}+\tau\left(\mathbf{q} u_{\delta}^{n}, \nabla u_{\delta}^{n}\right)+\left(v_{\delta}^{n}-v_{\delta}^{n-1}, u_{\delta}^{n}\right)=0 .
$$

We rewrite the left hand side to get

$$
\frac{1}{2}\left(\left\|u_{\delta}^{n}\right\|^{2}-\left\|u_{\delta}^{n-1}\right\|^{2}+\left\|u_{\delta}^{n}-u_{\delta}^{n-1}\right\|^{2}\right)+\tau\left\|\nabla u_{\delta}^{n}\right\|^{2} \leq\left\|v_{\delta}^{n}-v_{\delta}^{n-1}\right\|\left\|u_{\delta}^{n}\right\| \leq C \tau .
$$

Summing over $n$ we obtain

$$
\frac{1}{2}\left\|u_{\delta}^{N}\right\|^{2}+\frac{1}{2} \sum_{n=1}^{N}\left\|u_{\delta}^{n}-u_{\delta}^{n-1}\right\|^{2}+\tau \sum_{n=1}^{N}\left\|\nabla u_{\delta}^{n}\right\|^{2} \leq \frac{1}{2}\left\|u_{I}\right\|^{2}+C \leq C .
$$

leading to (3.7).

We proceed further to prove (3.8) and (3.9). We choose for the test function $\phi=u_{\delta}^{n}-u_{\delta}^{n-1}$ in (3.2) $)_{1}$ to obtain

$$
\left\|u_{\delta}^{n}-u_{\delta}^{n-1}\right\|^{2}+\tau\left(\nabla u_{\delta}^{n}, \nabla\left(u_{\delta}^{n}-u_{\delta}^{n-1}\right)\right)+\tau\left(\mathbf{q} u_{\delta}^{n}, \nabla\left(u_{\delta}^{n}-u_{\delta}^{n-1}\right)\right)+\left(v_{\delta}^{n}-v_{\delta}^{n-1}, u_{\delta}^{n}-u_{\delta}^{n-1}\right)=0 .
$$


Treating the terms on the left hand side separately

$$
\begin{aligned}
\tau\left(\nabla u_{\delta}^{n}, \nabla\left(u_{\delta}^{n}-u_{\delta}^{n-1}\right)\right) & =\tau \frac{1}{2}\left(\left\|\nabla u_{\delta}^{n}\right\|^{2}-\left\|\nabla u_{\delta}^{n-1}\right\|^{2}+\left\|\nabla\left(u_{\delta}^{n}-u_{\delta}^{n-1}\right)\right\|^{2}\right), \\
\tau\left|\left(\nabla \cdot\left(\mathbf{q} u_{\delta}^{n}\right),\left(u_{\delta}^{n}-u_{\delta}^{n-1}\right)\right)\right| & \leq \frac{\tau^{2} M_{q}^{2}\left\|\nabla u_{\delta}^{n}\right\|^{2}}{2}+\frac{1}{2}\left\|u_{\delta}^{n}-u_{\delta}^{n-1}\right\|^{2}, \\
\left|\left(v_{\delta}^{n}-v_{\delta}^{n-1}, u_{\delta}^{n}-u_{\delta}^{n-1}\right)\right| & \leq\left(\left\|v_{\delta}^{n}-v_{\delta}^{n-1}\right\|^{2}+\frac{1}{4}\left\|u_{\delta}^{n}-u_{\delta}^{n-1}\right\|^{2}\right) \leq \tau^{2} C M_{u}^{2}+\frac{1}{4}\left\|u_{\delta}^{n}-u_{\delta}^{n-1}\right\|^{2},
\end{aligned}
$$

where for the last line we have used (3.6). Using above, we obtain by summing over $n=1, \ldots, N$

$$
\frac{1}{4} \sum_{n=1}^{N}\left\|u_{\delta}^{n}-u_{\delta}^{n-1}\right\|^{2}+\tau\left\|\nabla u_{\delta}^{N}\right\|^{2}+\sum_{n=1}^{N} \tau\left\|\nabla\left(u_{\delta}^{n}-u_{\delta}^{n-1}\right)\right\|^{2} \leq C \tau+C M_{q}^{2} \tau+\tau\left\|\nabla u_{I}\right\|^{2} \leq C \tau .
$$

3.2 Convergence We consider the sequence of time discrete $\left(u_{\delta}^{n}, v_{\delta}^{n}, w_{\delta}^{n}\right)$ solving problem $\mathbf{P}_{\delta}^{n}$, and construct a time continuous approximation by taking linear interpolation. We define,

$$
\begin{array}{r}
U^{\tau}(t):=u_{\delta}^{n} \frac{\left(t-t_{n-1}\right)}{\tau}+u_{\delta}^{n-1} \frac{\left(t_{n}-t\right)}{\tau}, \\
V^{\tau}(t):=v_{\delta}^{n} \frac{\left(t-t_{n-1}\right)}{\tau}+v_{\delta}^{n-1} \frac{\left(t_{n}-t\right)}{\tau}, \\
W^{\tau}(t):=H_{\delta}\left(V^{\tau}(t)\right) .
\end{array}
$$

We will use compactness arguments for time-continuous triples $\left(U^{\tau}, V^{\tau}, W^{\tau}\right)$ to identify the limit points and the system that these limit points satisfy. Note that this triple depends on $\delta$ however, for notational convenience we have suppressed the subscript. Using the estimates obtained in (3.5)-(3.9), we obtain similar estimates for $\left(U^{\tau}, V^{\tau}, W^{\tau}\right)$.

LEMMA 3.6. We have the following estimates:

$$
\begin{array}{r}
0 \leq U^{\tau} \leq M_{u}, 0 \leq V^{\tau} \leq M_{v} e^{C T}, 0 \leq W^{\tau} \leq 1 \\
\left\|U^{\tau}\right\|^{2}+\left\|V^{\tau}\right\|^{2} \leq C, \\
\left\|\partial_{t} U^{\tau}\right\|^{2}+\left\|\nabla U^{\tau}\right\|^{2}+\left\|\partial_{t} V^{\tau}\right\|^{2} \leq C,
\end{array}
$$

where $C$ is a constant independent of $\tau, \delta$.

Proof. Clearly, (3.10), (3.11) follow from $L^{\infty}$ estimates for $u_{\delta}^{n}, v_{\delta}^{n}$.

We proceed with the gradient estimates. We rewrite the interpolation scheme,

$$
U^{\tau}=u_{\delta}^{n-1}+\frac{t-t_{n-1}}{\tau}\left(u_{\delta}^{n}-u_{\delta}^{n-1}\right)
$$

from which we obtain

$$
\nabla U^{\tau}=\nabla u_{\delta}^{n-1}+\frac{t-t_{n-1}}{\tau}\left(\nabla u_{\delta}^{n}-\nabla u_{\delta}^{n-1}\right) .
$$

Computing the $L^{2}(\Omega)$ norm and using the elementary inequality,

$$
\left\|\nabla U^{\tau}\right\|^{2} \leq 2\left\|\nabla u_{\delta}^{n-1}\right\|^{2}+2 \frac{\left(t-t_{n-1}\right)^{2}}{\tau^{2}}\left\|\nabla\left(u_{\delta}^{n}-u_{\delta}^{n-1}\right)\right\|^{2},
$$

and integrating over $t$ and since $u_{\delta}^{n-1}, u_{\delta}^{n}$ are constant in $\left(t_{n-1}, t_{n}\right)$ we obtain

$$
\begin{aligned}
\int_{0}^{T}\left\|\nabla U^{\tau}\right\|^{2} d t & \leq \sum_{n=1}^{N} 2 \int_{t_{n-1}}^{t_{n}}\left\|\nabla u_{\delta}^{n-1}\right\|^{2} d t+2 \sum_{n=1}^{N} \int_{t_{n-1}}^{t_{n}} \frac{\left(t-t_{n-1}\right)^{2}}{\tau^{2}}\left\|\nabla\left(u_{\delta}^{n}-u_{\delta}^{n-1}\right)\right\|^{2} d t \\
& \leq \sum_{n=1}^{N} 2 \tau\left\|\nabla u_{\delta}^{n-1}\right\|^{2}+\sum_{n=1}^{N} \frac{2 \tau}{3}\left\|\nabla\left(u_{\delta}^{n}-u_{\delta}^{n-1}\right)\right\|^{2}
\end{aligned}
$$


and stability estimates (3.7), (3.9) imply

$$
\left\|\nabla U^{\tau}\right\|_{L^{2}\left(\Omega^{T}\right)}^{2} \leq C
$$

To estimate $\left\|\partial_{t} V^{\tau}\right\|_{L^{2}\left(\Omega^{T}\right)}$ we note that whenever $t \in\left(t_{n-1}, t_{n}\right]$

$$
\partial_{t} V^{\tau}=\frac{v_{\delta}^{n}-v_{\delta}^{n-1}}{\tau}
$$

implying

$$
\int_{0}^{T}\left\|\partial_{t} V^{\tau}\right\|^{2} d t=\sum_{n=1}^{N} \int_{t_{n-1}}^{t_{n}}\left\|\frac{v_{\delta}^{n}-v_{\delta}^{n-1}}{\tau}\right\|^{2} d t \leq \sum_{n=1}^{N} \tau\left\|\frac{v_{\delta}^{n}-v_{\delta}^{n-1}}{\tau}\right\|^{2} \leq C N \tau \leq C T \leq C,
$$

where we have used the estimate (3.6). To prove the estimate (3.12) is similar as above. Again, for $\left\|\partial_{t} U^{\tau}\right\|_{L^{2}\left(0, T ; L^{2}(\Omega)\right)}^{2}$ term, we note that whenever $t \in\left(t_{n-1}, t_{n}\right]$

$$
\partial_{t} U^{\tau}=\frac{u_{\delta}^{n}-u_{\delta}^{n-1}}{\tau}
$$

of which square of $L^{2}$ norm upon integrating over $t$ yields

$$
\int_{0}^{T}\left\|\partial_{t} U^{\tau}\right\|^{2} d t=\sum_{n=1}^{N} \int_{t_{n-1}}^{t_{n}}\left\|\frac{u_{\delta}^{n}-u_{\delta}^{n-1}}{\tau}\right\|^{2} d t \leq \sum_{n=1}^{N} \tau\left\|\frac{u_{\delta}^{n}-u_{\delta}^{n-1}}{\tau}\right\|^{2} \leq C N \tau \leq C T \leq C,
$$

where we have used the estimate (3.8). This proves the lemma. $\square$

The next lemma provides the convergence results based on the estimates obtained above.

LEMMA 3.7. The estimates obtained are uniform in $\tau$ and $\delta$ and furthermore we have $\left(U^{\tau}, V^{\tau}, W^{\tau}\right) \in$ $\mathcal{U} \times \mathcal{V} \times L^{\infty}(\Omega)$. Clearly, $\tau \searrow 0$ with $\delta=O\left(\tau^{\frac{1}{2}}\right)$ implies that both $\delta, \frac{\tau}{\delta} \searrow 0$. The compactness arguments from the bounds established in Lemma 3.6 lead to the following convergence results

1. $U^{\tau} \rightarrow u$ weakly in $L^{2}\left((0, T) ; H_{0}^{1}(\Omega)\right)$,

2. $\partial_{t} U^{\tau} \rightarrow \partial_{t} u$ weakly in $L^{2}\left((0, T) ; H^{-1}(\Omega)\right)$,

3. $V^{\tau} \rightarrow v$ weakly in $L^{2}\left((0, T) ; L^{2}(\Omega)\right)$,

4. $\partial_{t} V^{\tau} \rightarrow \partial_{t} v$ weakly in $L^{2}\left((0, T) ; L^{2}(\Omega)\right)$,

5. $W^{\tau} \rightarrow w$ weakly-star in $L^{\infty}(\Omega)$.

The compactness results above only provides the existence of these limit points and it remains to be shown that these points satisfy the weak formulation in the sense of Definition 2.1. In what follows, we proceed to prove the same.

Notice that the weak convergence for $U^{\tau}$ in $L^{2}\left((0, T) ; H_{0}^{1}(\Omega)\right)$ together with $\partial_{t} U^{\tau} \rightarrow \partial_{t} u$ weakly in $L^{2}\left((0, T) ; H^{-1}(\Omega)\right)$ imply

$$
U^{\tau} \rightarrow u \quad \text { strongly in } \quad L^{2}\left((0, T) ; L^{2}(\Omega)\right)
$$

The lemma above provides only a weak convergence for $V^{\tau}$ and in the wake of nonlinearities, to facilitate getting the limit equations, we improve the convergence for $V^{\tau}$ and show that under $H^{1}$ regularity of the initial datum, we obtain strong convergence. This is achieved by the translation estimates.

Fixing a $t \in\left(t_{n-1}, t_{n}\right](0<n \leq N)$ we define $\triangle_{\xi}$ as the translation operator

$$
\triangle_{\xi} f(y, t)=f(y, t)-f(y+\xi, t)
$$

We have the following lemma that provides the required translation estimate.

LEMMA 3.8. If $v_{I} \in H^{1}(\Omega)$ then the following estimate holds

$$
\left\|\triangle_{\xi} v_{\delta}^{N}\right\|^{2}+\sum_{n=1}^{N}\left\|\triangle_{\xi}\left(v_{\delta}^{n}-v_{\delta}^{n-1}\right)\right\|^{2} \leq C\left\|\triangle_{\xi} v_{I}\right\|^{2}+C \tau \sum_{n=1}^{N}\left\|\triangle_{\xi} u_{\delta}^{n}\right\|^{2}
$$


Proof. For $\theta=\triangle_{\xi} v_{\delta}^{n}$ in (3.2) 2 , we get

$$
\left(\triangle_{\xi} v_{\delta}^{n}-\triangle_{\xi} v_{\delta}^{n-1}, \triangle_{\xi} v_{\delta}^{n}\right)=\tau\left(\triangle_{\xi} r\left(u_{\delta}^{n}\right), \triangle_{\xi} v_{\delta}^{n}\right)-\tau\left(\triangle_{\xi} H_{\delta}\left(v_{\delta}^{n-1}\right), \triangle_{\xi} v_{\delta}^{n}\right)
$$

We rewrite the last term in the above identity,

$$
\left(\triangle_{\xi} H_{\delta}\left(v_{\delta}^{n-1}\right), \triangle_{\xi} v_{\delta}^{n}\right)=\left(\triangle_{\xi} H_{\delta}\left(v_{\delta}^{n-1}\right), \triangle_{\xi} v_{\delta}^{n-1}\right)+\left(\triangle_{\xi} H_{\delta}\left(v_{\delta}^{n-1}\right), \triangle_{\xi}\left(v_{\delta}^{n}-v_{\delta}^{n-1}\right)\right) .
$$

The monotonicity of $H_{\delta}$ implies that the first term on the right hand side is positive, that is,

$$
\left(\triangle_{\xi} H_{\delta}\left(v_{\delta}^{n-1}\right), \triangle_{\xi} v_{\delta}^{n}\right) \geq 0 .
$$

For the left hand side, we use the identity

$$
\left(\triangle_{\xi} v_{\delta}^{n}-\triangle_{\xi} v_{\delta}^{n-1}, \triangle_{\xi} v_{\delta}^{n}\right)=\frac{1}{2}\left(\left\|\triangle_{\xi} v_{\delta}^{n}\right\|^{2}-\left\|\triangle_{\xi} v_{\delta}^{n-1}\right\|^{2}+\left\|\triangle_{\xi}\left(v_{\delta}^{n}-v_{\delta}^{n-1}\right)\right\|^{2}\right) .
$$

Using the above identity and Cauchy-Schwarz for the first term on the right hand side, we have

$$
\begin{array}{r}
\frac{1}{2}\left(\left\|\triangle_{\xi} v_{\delta}^{n}\right\|^{2}-\left\|\triangle_{\xi} v_{\delta}^{n-1}\right\|^{2}+\left\|\triangle_{\xi}\left(v_{\delta}^{n}-v_{\delta}^{n-1}\right)\right\|^{2}\right) \leq \tau L_{r}\left\|\triangle_{\xi} u_{\delta}^{n}\right\|\left\|\triangle_{\xi} v_{\delta}^{n}\right\| \\
+\tau\left(\triangle_{\xi} H_{\delta}\left(v_{\delta}^{n-1}\right), \triangle_{\xi}\left(v_{\delta}^{n}-v_{\delta}^{n-1}\right)\right) \\
\leq L_{r} \frac{\tau}{2}\left\|\triangle_{\xi} u_{\delta}^{n}\right\|^{2}+\frac{\tau}{2}\left\|\triangle_{\xi} v_{\delta}^{n}\right\|^{2}+\tau^{2}\left\|\triangle_{\xi} H_{\delta}\left(v_{\delta}^{n-1}\right)\right\|^{2}+\frac{1}{4}\left\|\triangle_{\xi}\left(v_{\delta}^{n}-v_{\delta}^{n-1}\right)\right\|^{2}
\end{array}
$$

which gives further

$$
\begin{array}{r}
\frac{1}{2}\left(\left\|\triangle_{\xi} v_{\delta}^{n}\right\|^{2}-\left\|\triangle_{\xi} v_{\delta}^{n-1}\right\|^{2}+\left\|\triangle_{\xi}\left(v_{\delta}^{n}-v_{\delta}^{n-1}\right)\right\|^{2}\right) \leq \frac{1}{2} L_{r} \tau\left\|\triangle_{\xi} u_{\delta}^{n}\right\|^{2}+\frac{1}{2} \tau\left\|\triangle_{\xi} v_{\delta}^{n}\right\|^{2} \\
+\frac{\tau^{2}}{\delta^{2}}\left\|\triangle_{\xi} v_{\delta}^{n-1}\right\|^{2}+\frac{1}{4}\left\|\triangle_{\xi}\left(v_{\delta}^{n}-v_{\delta}^{n-1}\right)\right\|^{2} .
\end{array}
$$

Summing over $n=1, \ldots, N$

$$
\begin{array}{r}
\left\|\triangle_{\xi} v_{\delta}^{N}\right\|^{2}+\frac{1}{2} \sum_{n=1}^{N}\left\|\triangle_{\xi}\left(v_{\delta}^{n}-v_{\delta}^{n-1}\right)\right\|^{2} \leq\left\|\triangle_{\xi} v_{I}\right\|^{2}+L_{r} \tau \sum_{n=1}^{N}\left\|\triangle_{\xi} u_{\delta}^{n}\right\|^{2} \\
+\tau \sum_{n=1}^{N}\left\|\triangle_{\xi} v_{\delta}^{n}\right\|^{2}+\sum_{n=1}^{N} \frac{2 \tau^{2}}{\delta^{2}}\left\|\triangle_{\xi} v_{\delta}^{n-1}\right\|^{2} .
\end{array}
$$

Choose for $\delta=O(\sqrt{\tau})$ and using Gronwall's lemma we obtain (since we can re-do the steps for any chosen $\left.k \leq N, k \in \mathbb{Z}_{+}\right)$

$$
\sup _{k=1, \ldots, N}\left\|\triangle_{\xi} v_{\delta}^{k}\right\|^{2} \leq C \tau \sum_{n=1}^{N}\left\|\triangle_{\xi} u_{\delta}^{n}\right\|^{2}+\left\|\triangle_{\xi} v_{I}\right\|^{2} .
$$

Using the translation estimate in above Lemma 3.8, we now show the strong convergence of $V^{\tau}$.

LEMMA 3.9. It holds that

$$
V^{\tau} \rightarrow v \quad \text { strongly in } \quad L^{2}\left(0, T ; L^{2}(\Omega)\right) .
$$

Proof. We use the translation in space and time to prove this strong convergence. First, let $\Omega^{\prime}$ denote an arbitrary compact subset of $\Omega$ and $\xi \in\left(0, \operatorname{dist}\left(\Gamma, \Omega^{\prime}\right)\right)$. It is well known that the strong convergence is tantamount to proving that ( Prop. 9.3, p.267, [4])

$$
\mathcal{I}:=\int_{\Omega^{\prime} T}\left|V^{\tau}\left(t+h_{t}, x+\xi\right)-V^{\tau}(t, x)\right|^{2} d x d t \searrow 0 \quad \text { as } \quad\left|\left(h_{t}, \xi\right)^{\prime}\right| \searrow 0 .
$$

Since $\partial_{t} V^{\tau}$ is bounded uniformly in $L^{2}\left(0, T ; L^{2}(\Omega)\right)$, the estimates for the translation with respect to time is easily obtained. We now consider the translation with respect to space. We have using the definition of $V^{\tau}$,

$$
\triangle_{\xi} V^{\tau}=\frac{t_{n}-t}{\tau} \triangle_{\xi} v_{\delta}^{n-1}+\frac{t-t_{n-1}}{\tau} \triangle_{\xi} v_{\delta}^{n}
$$


We define the piecewise constant interpolation $\bar{U}^{n}$ for $u_{\delta}^{n}$ so that

$$
\bar{U}^{n}=u_{\delta}^{n}, \quad t \in\left[t_{n-1}, t_{n}\right) .
$$

Squaring and integrating the left hand side over $\Omega^{\prime} \times(0, T)$, we obtain

$$
\int_{0}^{T} \int_{\Omega^{\prime}}\left|\triangle_{\xi} V^{\tau}\right|^{2} \leq \sum_{n=1}^{N}\left\|\triangle_{\xi} v_{\delta}^{n}\right\|^{2}
$$

and using (3.17) and replacing $u_{\delta}^{n}$ by $\bar{U}^{n}$, the right hand side is estimated as

$$
\int_{\Omega^{\prime}}\left|\triangle_{\xi} V^{\tau}(t, x) d x\right|^{2} \leq C T \tau \sum_{n=1}^{N}\left\|\triangle_{\xi} \bar{U}^{n}\right\|^{2}+C T\left\|\triangle_{\xi} v_{I}\right\|^{2}
$$

We exploit a general result (for example, see [22]) which connects the convergence of piecewise linear interpolations with that of the piecewise constant interpolations. This ensures that the strong convergence of affine interpolation $U^{\tau}$ in $L^{2}\left(0, T ; L^{2}(\Omega)\right)$ implies strong convergence for piece-wise constant interpolation $\bar{U}^{n}$. Having this strong convergence for $\bar{U}^{n}$ implies that the space translation is controlled, namely

$$
\tau \sum_{n=1}^{N}\left\|\triangle_{\xi} \bar{U}^{n}\right\|^{2} \searrow 0 \quad \text { as } \quad|\xi| \searrow 0 \text {. }
$$

Since the initial conditions are in $H^{1}(\Omega),\left\|\triangle_{\xi} v_{I}\right\|^{2} \searrow 0$ as $|\xi| \searrow 0$. With this, $\mathcal{I} \searrow 0$ as $|\xi| \searrow 0$ which leads to the strong convergence of $V^{\tau}$.

With the strong convergence established for $V^{\tau}$, our preparation is complete to show that the limits points $(u, v, w)$ obtained in Lemma 3.7 satisfy the weak formulation (2.7). This is considered in the following theorem.

THEOREM 3.10. The limit triple $(u, v, w)$ satisfy the weak formulation stated in Definition 2.1.

Proof. By the weak convergence, the estimates in Lemma 3.6 carry over for the limit triple $(u, v, w)$. Moreover,

$$
\begin{array}{r}
\int_{0}^{T}\left(\partial_{t} U^{\tau}, \phi\right) d t+\int_{0}^{T}\left(\nabla U^{\tau}, \nabla \phi\right) d t+\int_{0}^{T}\left(\mathbf{q} \cdot \nabla U^{\tau}, \phi\right) d t+\int_{0}^{T}\left(\partial_{t} V^{\tau}, \phi\right) d t \\
=\sum_{n=1}^{N} \int_{t_{n-1}}^{t_{n}}\left(\left(\nabla U^{\tau}-\nabla u_{\delta}^{n}\right), \nabla \phi\right) d t+\sum_{n=1}^{N} \int_{t_{n-1}}^{t_{n}}\left(\mathbf{q} \cdot\left(\nabla U^{\tau}-\nabla u_{\delta}^{n}\right), \phi\right) d t
\end{array}
$$

for all $\phi \in L^{2}\left(0, T ; H_{0}^{1}\right)$. By Lemma 3.7, the left hand side converges to the desired limit. We are thus required to show that the right hand side vanishes. Denote the integrals of the right hand side by $\mathcal{I}_{1}$ and $\mathcal{I}_{2}$ and we obtain

$$
\left|\mathcal{I}_{1}\right| \leq\left(\sum_{n=1}^{N} \tau C\left\|\nabla u_{\delta}^{n}-\nabla u_{\delta}^{n-1}\right\|^{2}\right)^{\frac{1}{2}}\left(\int_{0}^{T}\|\nabla \phi\|^{2} d t\right)^{\frac{1}{2}} \rightarrow 0
$$

since $\sum_{n=1}^{N} \tau\left\|\nabla u_{\delta}^{n}-\nabla u_{\delta}^{n-1}\right\|^{2} \searrow 0$ because of estimate (3.9). Similarly,

$$
\left|\mathcal{I}_{2}\right| \leq\left(\sum_{n=1}^{N} \tau M_{q}\left\|\nabla\left(u_{\delta}^{n}-u_{\delta}^{n-1}\right)\right\|^{2}\right)^{\frac{1}{2}}\left(\int_{0}^{T}\|\phi\|^{2} d t\right)^{\frac{1}{2}}
$$

and due to (3.9), $\left|\mathcal{I}_{2}\right|$ vanishes as well.

For the limiting equation for $V^{\tau}$, we have using (3.2)

$$
\begin{array}{r}
\int_{0}^{T}\left(\partial_{t} V^{\tau}, \theta\right) d t=\int_{0}^{T}\left(r\left(U^{\tau}\right)-W^{\tau}\right) d t+\sum_{n=1}^{N} \int_{t_{n-1}}^{t_{n}}\left(r\left(u_{\delta}^{n}\right)-r\left(U^{\tau}\right), \theta\right) d t \\
+\sum_{n=1}^{N} \int_{t_{n-1}}^{t_{n}}\left(W^{\tau}-H_{\delta}\left(v_{\delta}^{n-1}\right), \theta\right) d t .
\end{array}
$$


We would retrieve the desired limiting equations once we prove that the last two integrals on the right hand side vanish. Let us denote the last two integrals by $\mathcal{I}_{3}$ and $\mathcal{I}_{4}$ respectively. We have

$$
\left|\mathcal{I}_{3}\right| \leq\left(\sum_{n=1}^{N} \tau L_{r}^{2}\left\|u_{\delta}^{n}-u_{\delta}^{n-1}\right\|^{2}\right)^{\frac{1}{2}}\left(\int_{0}^{T}\|\theta\|^{2} d t\right)^{\frac{1}{2}}
$$

and using (3.8), we obtain $\mathcal{I}_{3} \searrow 0$.

For $\mathcal{I}_{4}$, use the definition of $W^{\tau}$ and Lipschitz continuity of $H_{\delta}$ in order to obtain

$$
\left|\mathcal{I}_{4}\right| \leq \sum_{n=1}^{N} \int_{t_{n-1}}^{t_{n}} \frac{1}{\delta}\left\|v_{\delta}^{n}-v_{\delta}^{n-1}\right\|\|\theta\| d t \leq \sum_{n=1}^{N} C \tau \frac{\tau}{\delta}\|\theta\| d t
$$

by using (3.5) and further using Cauchy Schwarz and $\tau / \delta \searrow 0$ (by the construction of $\delta$ ) we obtain $\mathcal{I}_{4} \searrow 0$.

Next, we need to prove $w=H(v)$. Since we have $V^{\tau}$ strongly converging, we also obtain $V^{\tau} \rightarrow v$ pointwise a.e.. Further,

$$
\left(W^{\tau}, \theta\right)=(w, \theta)
$$

and by definition, $W^{\tau}=H_{\delta}\left(V^{\tau}\right)$; and

$$
\left(H_{\delta}\left(V^{\tau}\right), \theta\right)=(w, \theta) .
$$

By construction, as $\tau \searrow 0, \delta \searrow 0$. Now, for any given $(t, x) \in \Omega$, either $v>0$ or $v=0$. For the case when $v>0$, we have $W^{\tau}=1$ and hence $w=1$. For the case when $v=0$, since $\partial_{t} v \in L^{2}(\Omega), \partial_{t} v=0$ leading to $w=r(u)$ with $0 \leq w \leq 1$ and hence we can establish the required result. $\square$

4 Fully discrete system We consider the fully discrete system (discretized in both space and time) and show the convergence of the numerical method. In particular, we consider the finite element discretization in space and for the time we retain the discretization as in the semi-discrete case. The steps for the proof of convergence are similar to the semi-discrete situation and where ever the proof is based on the time-discrete case treated above, we suppress the details of the proof. Further, to simplify notation, henceforth, we suppress the subscript $\delta$.

To obtain the fully discrete formulation for the weak solution of (2.1)-(2.2), we take Euler's implicit discretization for the diffusion and reaction terms. Next, with $N \in \mathbb{N}, \tau=\frac{T}{N}$ and $t_{n}=n \tau, n=1, \ldots, N$, we consider a uniform time stepping that is implicit in $u$ and explicit in $v$. For the space discretization, we have $\Omega$ discretized in the $2-$ dimensional simplices (triangles) $\mathcal{T}_{h}$ with mesh-size $h$. We have assumed $\Omega$ to be polygonal as has been stated in Section 2.1. Further, we give definitions, in Section 2.1, of function spaces that will be used here. Starting with $u_{h}^{0}=u_{I}, v_{h}^{0}=v_{I}$, with $n \in\{1, \ldots, N\}$, the approximation $\left(u_{h}^{n}, v_{h}^{n}\right)$ of $\left(u\left(t_{n}\right), v\left(t_{n}\right)\right)$ at $\mathcal{T}_{h}$ solves

Problem $\mathbf{P}_{h}^{n}$ : Given $\left(u_{h}^{n-1}, v_{h}^{n-1}, w_{h}^{n-1}\right) \in \mathcal{U}_{h} \times \mathcal{U}_{h} \times L^{\infty}(\Omega)$, find $\left(u_{h}^{n}, v_{h}^{n}, w_{h}^{n}\right) \in \mathcal{U}_{h} \times \mathcal{U}_{h} \times L^{\infty}(\Omega)$ such that

$$
\begin{array}{r}
\left(u_{h}^{n}-u_{h}^{n-1}, \phi_{h}\right)+\tau\left(\nabla u_{h}^{n}, \nabla \phi_{h}\right)-\left(\mathbf{q}_{h} u_{h}^{n}, \nabla \phi_{h}\right)+\left(v_{h}^{n}-v_{h}^{n-1}, \phi_{h}\right)=0, \\
\left(v_{h}^{n}-v_{h}^{n-1}, \theta_{h}\right)=\tau\left(r\left(u_{h}^{n}\right), \theta_{h}\right)-\tau\left(H_{\delta}\left(v_{h}^{n-1}\right), \theta_{h}\right)
\end{array}
$$

for all $\phi_{h}, \theta_{h} \in \mathcal{U}_{h}$.

To complete the solution we define

$$
w_{h}^{n}:=H_{\delta}\left(v_{h}^{n-1}\right) .
$$

Note that the solutions for $\mathbf{P}_{h}^{n}$ are defined in a finite dimensional vector space. In what follows we analyse the fully discrete case. 
4.1 Existence and uniqueness The discretization above in (4.1) provides a sequence of elliptic equations for $u_{h}^{n}, v_{h}^{n}$ given $u_{h}^{n-1}, v_{h}^{n-1} \in \mathcal{U}_{h}$. For stability reasons, we choose $\delta=O\left(\tau^{\frac{1}{2}}\right)$.

The first task here is to obtain the existence and uniqueness for problem $\mathbf{P}_{h}^{n}$. Since $\mathbf{P}_{h}^{n}$ is defined on a finite dimensional space, the proof for existence and uniqueness is not difficult, as for example it follows from [36]. However, we present here a fixed point argument based on linearization techniques (see [29]). It also provides a numerical method in addition to providing the existence and uniqueness. We use this method to perform numerical computations, hence we find it relevant to present this argument.

We assume that $u_{h}^{n-1}$ and $v_{h}^{n-1}$ are given with their corresponding $H_{0}^{1}(\Omega)$ and $L^{2}(\Omega)$ norms uniformly bounded. To construct the iteration scheme, we decouple the ion-transport equation and rewrite (4.1) using $(4.1)_{2}$

$$
\left(u_{h}^{n}-u_{h}^{n-1}, \phi_{h}\right)+\tau\left(\nabla u_{h}^{n}, \nabla \phi_{h}\right)-\tau\left(\mathbf{q}_{h} u_{h}^{n}, \nabla \phi_{h}\right)+\tau\left(r\left(u_{h}^{n}\right)-H_{\delta}\left(v_{h}^{n-1}\right), \phi_{h}\right)=0
$$

for all $\phi_{h} \in \mathcal{U}_{h}$.

We make some preparation for applying the fixed point iteration. We define the norm

$$
\|\| u\|\|^{2}:=\|u\|^{2}+\frac{2 \tau}{2+\tau L_{r}}\|\nabla u\|^{2}
$$

so that we can obtain the contraction. Recall that $L_{r}$ is the Lipschitz constant of the precipitation term $r(\cdot)$. Further, we define the closed set

$$
\mathcal{K}:=\left\{u \in \mathcal{U}_{h}, \quad\|u\| \mid \| C\right\} .
$$

Assuming $u_{h}^{n-1} \in \mathcal{K}$, we define the mapping $\mathcal{T}$

$$
u_{i-1} \mapsto u_{i}=\mathcal{T} u_{i-1}
$$

where $u_{i}$ solves

$$
\begin{aligned}
\left(u_{i}, \phi_{h}\right)+\tau\left(\nabla u_{i}, \nabla \phi_{h}\right)-\tau\left(\mathbf{q}_{h} u_{i}, \nabla \phi_{h}\right)+\tau L_{r}\left(u_{i}, \phi_{h}\right) & =\tau L_{r}\left(u_{i-1}, \phi_{h}\right)-\tau\left(r\left(u_{i-1}\right), \phi_{h}\right) \\
& +\left(u_{h}^{n-1}, \phi_{h}\right)+\tau\left(H_{\delta}\left(v_{h}^{n-1}\right), \phi_{h}\right)
\end{aligned}
$$

for all $\phi_{h} \in \mathcal{U}_{h}$. A good initial value for the iteration is $u_{0}=u_{h}^{n-1}$. We have suppressed the superscript $n$ in the iteration for the sake of presentation. In the iterative scheme considered here, we note that the fixed point satisfies (4.2) as the terms involving $L_{r}$ gets canceled in the case of convergence. The problem of proving existence and uniqueness of solution of problem $\mathbf{P}_{h}^{n}$ is, thus, tantamount to proving that of the fixed point of (4.3). Such constructions are common and we refer to [10] and the references therein for another application.

Next, we define

$$
e_{i}:=u_{i}-u_{i-1}
$$

We have the following result for the mapping $\mathcal{T}$.

LEMMA 4.1. It holds that $\mathcal{T}$ maps $u_{i-1} \in \mathcal{K}$ to $u_{i}=\mathcal{T} u_{i-1} \in \mathcal{K}$ and is a contraction with respect to the $|\| \cdot|||$ norm.

Proof. Note that standard Lax-Milgram arguments provide that $\mathcal{T}$ maps $\mathcal{K}$ into $H^{1}$. Next, we show that it is a contraction and hence maps $\mathcal{K}$ to itself. Using (4.3) and subtract the equation for $u_{i-1}$ to obtain

$$
\begin{aligned}
\left(e_{i}, \phi_{h}\right)+\tau\left(\nabla e_{i}, \nabla \phi_{h}\right)-\tau\left(\mathbf{q}_{h} e_{i}, \nabla \phi_{h}\right)+\tau L_{r}\left(e_{i}, \phi_{h}\right) & =\tau L_{r}\left(e_{i-1}, \phi_{h}\right) \\
& -\tau\left(r\left(u_{i-1}\right)-r\left(u_{i-2}\right), \phi_{h}\right) .
\end{aligned}
$$

Choosing for $\phi_{h}=e_{i}$, we obtain

$$
\left(1+\tau L_{r}\right)\left\|e_{i}\right\|^{2}+\tau\left\|\nabla e_{i}\right\|^{2}-\tau\left(\mathbf{q}_{h} e_{i}, \nabla e_{i}\right)=\tau\left(L_{r} e_{i-1}-r\left(u_{i-1}\right)+r\left(u_{i-2}\right), e_{i}\right)
$$


and since $r$ is Lipschitz continuous, we use mean value theorem to obtain

$$
\left(1+\tau L_{r}\right)\left\|e_{i}\right\|^{2}+\tau\left\|\nabla e_{i}\right\|^{2}-\tau\left(\mathbf{q}_{h} e_{i}, \nabla \phi\right)=\tau\left(L_{r} e_{i-1}-r^{\prime}(\xi) e_{i-1}, e_{i}\right)
$$

for some $\xi \in\left(u_{i-1}, u_{i-2}\right)$. Further, note that the contribution due to convection term vanishes, using the divergence free $\mathbf{q}_{h}$ we have

$$
\left(\mathbf{q}_{h} e_{i}, \nabla e_{i}\right)=\frac{1}{2} \int_{\Omega} \nabla \cdot\left(\mathbf{q}_{h} e_{i}^{2}\right) d x=\frac{1}{2} \int_{\partial \Omega} \nu \cdot \mathbf{q}_{h} e_{i}^{2} d s=0
$$

because of boundary condition for $e_{i}$.

Next, with $0 \leq L_{r}-r^{\prime}(\xi) \leq L_{r}$, the right hand side of (4.6) can be estimated as

$$
\left(1+\tau L_{r}\right)\left\|e_{i}\right\|^{2}+\tau\left\|\nabla e_{i}\right\|^{2} \leq \tau L_{r}\left\|e_{i-1}\right\|\left\|e_{i}\right\| \leq \frac{1}{2} \tau L_{r}\left(\left\|e_{i}\right\|^{2}+\left\|e_{i-1}\right\|^{2}\right)
$$

which gives,

$$
\left(1+\frac{1}{2} \tau L_{r}\right)\left\|e_{i}\right\|^{2}+\tau\left\|\nabla e_{i}\right\|^{2} \leq \frac{1}{2} \tau L_{r}\left\|e_{i-1}\right\|^{2}
$$

or rewriting

$$
\left\|e_{i}\right\|^{2}+\frac{\tau}{1+\frac{1}{2} \tau L_{r}}\left\|\nabla e_{i}\right\|^{2} \leq \frac{1}{2} \frac{\tau L_{r}}{1+\frac{1}{2} \tau L_{r}}\left\|e_{i-1}\right\|^{2} \leq \gamma\|\| e_{i-1}\|\|^{2}
$$

with $\gamma=\frac{\tau L_{r}}{2+\tau L_{r}}$ and using $\tau L_{r}>0$ we obtain $\gamma<1$. Hence, the map $\mathcal{T}$

$$
u_{i-1} \mapsto u_{i}=\mathcal{T} u_{i-1}
$$

is a contraction and therefore has a unique fixed point by Banach fixed point theorem.

Using the above lemma, we immediately obtain that $u_{i}$ converges to the fixed point of (4.2) as $i \nearrow \infty$. Clearly, the limit is $u_{h}^{n}$; after obtaining it, computing $v_{h}^{n}$ is straightforward as it is an explicit discretization in time. In other words,

LeMma 4.2. Problem $\boldsymbol{P}_{h}^{n}$ has a unique solution triple $\left(u_{h}^{n}, v_{h}^{n}, w_{h}^{n}\right)$.

REMARK 4.1. The numerical scheme presented here has a linear convergence rate with respect to $H^{1}$ norm as opposed to say Newton's iteration which is quadratic. This is compensated by the fact that the convergence is guaranteed for any choice of parameters. The error converges to 0 as the iteration index approaches $\infty$. In practice, however, only 3-4 iterations are needed. Also, in $L^{2}$ norm, one expects a faster convergence since, as $\tau$ decreases the factor $\gamma$ tends to 0 and the norm defined for the mapping $\mathcal{T}$ is independent of $\tau$ for $L^{2}$ part of the norm.

We have not investigated the stability in time of this iteration process. For computing the solution, at each time step, by performing this iterative scheme, we accumulate the error. It can be shown that the error accumulated in time vanishes as $\tau$ tends to 0 . Since our focus is to show the existence and uniqueness of the solution for the discrete problem $\boldsymbol{P}_{h}^{n}$ we do not investigate this aspect. For a discussion on this issue, we refer to [10], Lemma 3.6.

4.2 A priori estimates We start with the a priori estimates for Problem $\mathbf{P}_{h}^{n}$. These are similar to estimates for parabolic equations but here restricted to discrete time steps. We have the following lemma: 
LEMMA 4.3. The following estimates hold

$$
\begin{array}{r}
\sup _{k=1, \ldots, N}\left\|u_{h}^{k}\right\|^{2} \leq C, \\
\tau \sum_{n=1}^{N}\left\|\nabla u_{h}^{n}\right\|^{2} \leq C, \\
\left\|v_{h}^{n}-v_{h}^{n-1}\right\| \leq C \tau, \\
\sup _{k=1, \ldots, N}\left\|v_{h}^{k}\right\| \leq C, \\
\sum_{n=1}^{N}\left\|u_{h}^{n}-u_{h}^{n-1}\right\|^{2} \leq C \tau, \\
\sum_{n=1}^{N}\left\|\nabla\left(u_{h}^{n}-u_{h}^{n-1}\right)\right\|^{2} \leq C,
\end{array}
$$

where $C$ is independent of $\tau$ and $\delta$.

Proof. These a priori estimates are similar to the estimates as derived in the proof of Lemma 3.5. However, the technique used for obtaining the maximum principle in the continuous case, does not apply here due to inadmissibility of the test function. We therefore, derive the estimates in the following manner: Consider $(4.1)_{1}$ and choose $\phi_{h}=u_{h}^{n}$ to obtain

$$
\left(u_{h}^{n}-u_{h}^{n-1}, u_{h}^{n}\right)+\tau\left(\nabla u_{h}^{n}, \nabla u_{h}^{n}\right)-\tau\left(\mathbf{q}_{h} u_{h}^{n}, \nabla u_{h}^{n}\right)+\left(v_{h}^{n}-v_{h}^{n-1}, u_{h}^{n}\right)=0,
$$

and use $(4.1)_{2}$ to replace the last term on the left hand side,

$$
\left(u_{h}^{n}-u_{h}^{n-1}, u_{h}^{n}\right)+\tau\left(\nabla u_{h}^{n}, \nabla u_{h}^{n}\right)+\tau\left(r\left(u_{h}^{n}\right), u_{h}^{n}\right)=\tau\left(\mathbf{q}_{h} u_{h}^{n}, \nabla u_{h}^{n}\right)-\tau\left(H_{\delta}\left(v_{h}^{n-1}\right), u_{h}^{n}\right) .
$$

Using the identity for the left hand side

$$
\left(u_{h}^{n}-u_{h}^{n-1}, u_{h}^{n}\right)=\frac{1}{2}\left(\left\|u_{h}^{n}\right\|^{2}-\left\|u_{h}^{n-1}\right\|^{2}+\left\|u_{h}^{n}-u_{h}^{n-1}\right\|^{2}\right)
$$

and for the right hand side, use Cauchy-Schwarz to obtain

$$
\frac{1}{2}\left(\left\|u_{h}^{n}\right\|^{2}-\left\|u_{h}^{n-1}\right\|^{2}+\left\|u_{h}^{n}-u_{h}^{n-1}\right\|^{2}\right)+\frac{1}{2} \tau\left\|\nabla u_{h}^{n}\right\|^{2}+\tau L_{r}\left\|u_{h}^{n}\right\|^{2} \leq \frac{1}{2} \tau M_{q}\left\|u_{h}^{n}\right\|^{2}+C \tau+\frac{1}{2} \tau\left\|u_{h}^{n}\right\|^{2},
$$

and sum over $n=1, \ldots, N$ to get

$$
\frac{1}{2}\left\|u_{h}^{N}\right\|^{2}+\frac{1}{2} \sum_{n=1}^{N}\left\|u_{h}^{n}-u_{h}^{n-1}\right\|^{2}+\frac{1}{2} \tau \sum_{n=1}^{N}\left\|\nabla u_{h}^{n}\right\|^{2}+L_{r} \tau \sum_{n=1}^{N}\left\|u_{h}^{n}\right\|^{2} \leq C \tau \sum_{n=1}^{N}\left\|u_{h}^{n}\right\|^{2}+C .
$$

Using Gronwall's lemma provides

$$
\sup _{k=1, \ldots, N}\left\|u_{h}^{k}\right\|^{2} \leq C
$$

which is (4.7). Use this in above to obtain

$$
\tau \sum_{n=1}^{N}\left\|\nabla u_{h}^{n}\right\|^{2} \leq C
$$

which is (4.8). We proceed further to prove (4.9) and (4.10). Choose for $\theta=v_{h}^{n}-v_{h}^{n-1}$ in (4.1) 2 and applying Cauchy-Schwarz inequality for the right hand side,

$$
\left\|v_{h}^{n}-v_{h}^{n-1}\right\|^{2} \leq \tau\left\|r\left(u_{h}^{n}\right)\right\|\left\|v_{h}^{n}-v_{h}^{n-1}\right\|+\tau\left\|H_{\delta}\left(v_{h}^{n-1}\right)\right\|\left\|v_{h}^{n}-v_{h}^{n-1}\right\|
$$


which implies using bound (4.7) for $u_{h}^{n}$,

$$
\left\|v_{h}^{n}-v_{h}^{n-1}\right\| \leq C \tau
$$

which is (4.9).

Next to prove (4.10), choose $\theta=v_{h}^{n}$ in (4.1) 2 to obtain

$$
\left(v_{h}^{n}-v_{h}^{n-1}, v_{h}^{n}\right)=\tau\left(r\left(u_{h}^{n}\right), v_{h}^{n}\right)-\tau\left(H_{\delta}\left(v_{h}^{n-1}\right), v_{h}^{n}\right) .
$$

The left hand side can be rewritten as

$$
\left(v_{h}^{n}-v_{h}^{n-1}, v_{h}^{n}\right)=\frac{1}{2}\left(\left\|v_{h}^{n}\right\|^{2}-\left\|v_{h}^{n-1}\right\|^{2}+\left\|v_{h}^{n}-v_{h}^{n-1}\right\|^{2}\right) .
$$

We rewrite the last term on the right hand side

$$
\left(H_{\delta}\left(v_{h}^{n-1}\right), v_{h}^{n}\right)=\left(H_{\delta}\left(v_{h}^{n-1}\right), v_{h}^{n-1}\right)-\left(H_{\delta}\left(v_{h}^{n-1}\right), v_{h}^{n-1}-v_{h}^{n}\right) .
$$

Substituting these in (4.10),

$\left\|v_{h}^{n}\right\|^{2}-\left\|v_{h}^{n-1}\right\|^{2}+\left\|v_{h}^{n}-v_{h}^{n-1}\right\|^{2}=2 \tau\left(r\left(u_{h}^{n}\right), v_{h}^{n}\right)-2\left(H_{\delta}\left(v_{h}^{n-1}\right), v_{h}^{n-1}\right)+2\left(H_{\delta}\left(v_{h}^{n-1}\right), v_{h}^{n-1}-v_{h}^{n}\right)$.

Since $H(\cdot)$ is monotone, $\left(H_{\delta}\left(v_{h}^{n-1}\right), v_{h}^{n-1}\right) \geq 0$ and further use Cauchy-Schwarz inequality to get

$$
\left\|v_{h}^{n}\right\|^{2}-\left\|v_{h}^{n-1}\right\|^{2}+\left\|v_{h}^{n}-v_{h}^{n-1}\right\|^{2} \leq 2 \tau C\left\|u_{h}^{n}\right\|\left\|v_{h}^{n}\right\|+2 \tau\left(H_{\delta}\left(v_{h}^{n-1}\right), v_{h}^{n}-v_{h}^{n-1}\right) .
$$

Now use Young's inequality for the terms on the right hand side to obtain

$$
\left\|v_{h}^{n}\right\|^{2}-\left\|v_{h}^{n-1}\right\|^{2}+\frac{1}{2}\left\|v_{h}^{n}-v_{h}^{n-1}\right\|^{2} \leq C \tau\left\|v_{h}^{n}\right\|^{2}+C \tau\left\|u_{h}^{n}\right\|^{2}+2 \tau^{2}\left\|H_{\delta}\right\|^{2} .
$$

Summing over $n=1, \ldots, N$ gives

$$
\begin{aligned}
\left\|v_{h}^{N}\right\|^{2}+\frac{1}{2} \sum_{n=1}^{N}\left\|v_{h}^{n}-v_{h}^{n-1}\right\|^{2} & \leq\left\|v_{I}\right\|^{2}+C \tau \sum_{n=1}^{N}\left\|v_{h}^{n}\right\|^{2}+C \tau \sum_{n=1}^{N}\left\|u_{h}^{n}\right\|^{2}+\sum_{n=1}^{N} 2 \tau^{2}\left\|H_{\delta}\right\|^{2} \\
& \leq \tau \sum_{n=1}^{N}\left\|v_{\delta}^{n}\right\|^{2}+C+C \tau
\end{aligned}
$$

where we have used bounds on $u_{h}^{n}$ and on initial data. Now use Gronwall's lemma to conclude (4.10).

The estimates (4.11) and (4.12) follow from the steps in the proof of (3.8) and (3.9). We omit the details.

4.3 Convergence As in the semi-discrete case, we consider the sequence of time discrete $\left(u_{h}^{n}, v_{h}^{n}, w_{h}^{n}\right)$ solving problem $\mathbf{P}_{h}^{n}$, and construct a time continuous approximation by taking linear interpolation. We define,

$$
\begin{aligned}
U_{h}^{\tau}(t) & :=u_{h}^{n} \frac{\left(t-t_{n-1}\right)}{\tau}+u_{h}^{n-1} \frac{\left(t_{n}-t\right)}{\tau}, \\
V_{h}^{\tau}(t) & :=v_{h}^{n} \frac{\left(t-t_{n-1}\right)}{\tau}+v_{h}^{n-1} \frac{\left(t_{n}-t\right)}{\tau}, \\
W_{h}^{\tau}(t) & :=H_{\delta}\left(V_{h}^{\tau}(t)\right) .
\end{aligned}
$$

We will use compactness arguments for time-continuous triples $\left(U_{h}^{\tau}, V_{h}^{\tau}, W_{h}^{\tau}\right)$ to identify the limit points and the system that these limit points satisfy. Using the estimates obtained in (4.9)-(4.12), we obtain similar estimates for $\left(U_{h}^{\tau}, V_{h}^{\tau}, W_{h}^{\tau}\right)$.

LEMMA 4.4. We have the following estimates:

$$
\begin{array}{r}
\left\|U_{h}^{\tau}\right\|^{2}+\left\|V_{h}^{\tau}\right\|^{2} \leq C, \\
\left\|\partial_{t} U_{h}^{\tau}\right\|^{2}+\left\|\nabla U_{h}^{\tau}\right\|^{2}+\left\|\partial_{t} V_{h}^{\tau}\right\|^{2} \leq C, \\
0 \leq W_{h}^{\tau} \leq 1
\end{array}
$$


where the norms are taken with respect to $L^{2}\left(0, T ; L^{2}(\Omega)\right)$ and $C$ is a constant independent of $\tau, \delta$.

Proof. Estimate (4.18) follows from the $L^{2}$ estimate for $u_{h}^{k}$ and $v_{h}^{k}$. For instance,

$$
\left\|U_{h}^{\tau}\right\|_{L^{2}\left(0, T ; L^{2}(\Omega)\right)}^{2} \leq 2 \tau \sum_{n=1}^{N}\left\|u_{h}^{n}\right\|^{2}+2 \tau \sum_{n=1}^{N}\left\|u_{h}^{n-1}\right\|^{2} \leq C N \tau \leq C
$$

using (4.7). Similarly the estimate for $V_{h}^{\tau}$ follows from (4.10). The other estimates follow the steps in semi-discrete case. We omit the details.

The estimates above provide us with the convergence results. We state this in the next lemma.

LEMMA 4.5. The estimates obtained are uniform in discretization parameters $(\tau, h)$ and regularization parameter $\delta$ and furthermore we have $\left(U_{h}^{\tau}, V_{h}^{\tau}, W_{h}^{\tau}\right) \in \mathcal{U} \times \mathcal{V} \times L^{\infty}(\Omega)$. Clearly, $\tau \searrow 0$ with $\delta=O(\sqrt{\tau})$, implies that both $\delta, \frac{\tau}{\delta} \searrow 0$. The compactness arguments from the Lemma 4.4 lead to the following convergence results. As $(h, \tau) \searrow 0$, it holds that

1. $U_{h}^{\tau} \rightarrow u$ weakly in $L^{2}\left((0, T) ; H_{0}^{1}(\Omega)\right)$,

2. $\partial_{t} U_{h}^{\tau} \rightarrow \partial_{t} u$ weakly in $L^{2}\left((0, T) ; L^{2}(\Omega)\right)$,

3. $V_{h}^{\tau} \rightarrow v$ weakly in $L^{2}\left((0, T) ; L^{2}(\Omega)\right)$,

4. $\partial_{t} V_{h}^{\tau} \rightarrow \partial_{t} v$ weakly in $L^{2}\left((0, T) ; L^{2}(\Omega)\right)$,

5. $W_{h}^{\tau} \rightarrow w$ weakly-star in $L^{\infty}(\Omega)$.

Once again, it remains to be proved that the limit points are the desired functions that satisfy the weak formulation in the sense of Definition 2.1. In this respect, to identify the limit object for the dissolution term, we need the strong convergence of $V_{h}^{\tau}$. Here, the translation estimates can not be applied straightforwardly as the test functions after translation may leave the space $\mathcal{U}_{h}$. This is due to the fact that the translations are not remaining within the same triangle. To prove the strong convergence, we use the ideas from [7] . In this context, we use the higher regularity of $v_{I}$ to improve the convergence. We start with the following proposition that we are going to use later.

PROPOSITION 4.6. Let $\Pi$ be the interpolation operator that maps $H^{1}(\Omega) \cap C(\bar{\Omega})$ to the space $\mathcal{U}_{h}$. Let $g: \mathbb{R} \mapsto \mathbb{R}$ be a Lipschitz function with Lipschitz constant $L_{g}$, and let $f: \Omega \mapsto \mathbb{R}$ defined by $f=g(u)$. Then for any $u \in \mathcal{U}_{h}$ it holds that

$$
\|\nabla \Pi f\| \leq L_{g}\|\nabla u\|
$$

Proof. We follow the computations of [7] (Page 469) and only provide a brief sketch and cite the results directly (also see [15]). Following the notations of [7], let $A_{i}^{T}=\left(x_{i}, y_{i}\right)$ denote the vertices of the triangle $T$ with $i=1,2$, or 3 . Note that $f$ is an $H^{1}$ function. Since $\Pi f$ is piecewise linear,

$$
\Pi f=\sum_{i=1}^{3}\left(a_{i}^{T} x+b_{i}^{T} y+c_{i}^{T}\right) \Pi f\left(A_{i}^{T}\right),
$$

with

$$
\begin{gathered}
a_{i}^{T}=\frac{1}{2|T|}\left(y_{j}-y_{k}\right), \\
b_{i}^{T}=\frac{1}{2|T|}\left(x_{k}-x_{j}\right), \\
c_{i}^{T}=\frac{1}{2|T|}\left(x_{j} y_{k}-x_{k} y_{j}\right)
\end{gathered}
$$

with cyclic permutation of the indices $i, j, k$ and $|T|$ representing the area of the triangle. The equation (2.5), page 469 of [7] reads for the given context here,

$$
\|\nabla \Pi f\|^{2}=\sum_{T \in \mathcal{T}_{h}} \frac{1}{4|T|}\left\{\left(A_{k}^{T} A_{i}^{T}, A_{k}^{T} A_{j}^{T}\right)\left|f\left(A_{i}^{T}\right)-f\left(A_{j}^{T}\right)\right|^{2}\right\}
$$


where $A_{k}^{T} A_{i}^{T}$ is a vector connecting vertex $i$ to $k$ of triangle $T$. This provides using the Lipschitz continuity of $g$,

$$
\begin{aligned}
\|\nabla \Pi f\|^{2} & \leq L_{g}^{2} \sum_{T \in \mathcal{T}_{h}} \frac{1}{4|T|}\left\{\left(A_{k}^{T} A_{i}^{T}, A_{k}^{T} A_{j}^{T}\right)\left|u\left(A_{i}^{T}\right)-u\left(A_{j}^{T}\right)\right|^{2}\right\} \\
& =L_{g}^{2}\|\nabla u\|^{2}
\end{aligned}
$$

establishing the proposition.

REMARK 4.2. It is important to note that the constant of the bound is indeed $L_{g}$, the Lipschitz constant. We need this fact below in the proof of the next Lemma.

LEMMA 4.7. If $v_{I} \in H^{1}(\Omega)$, it holds that as $(\tau, h) \searrow 0$

$$
V_{h}^{\tau} \rightarrow v \quad \text { strongly in } \quad L^{2}\left(0, T ; L^{2}(\Omega)\right) .
$$

Proof. From $(4.1)_{2}$ we have

$$
\left(v_{h}^{n}-v_{h}^{n-1}, \theta_{h}\right)=\tau\left(r\left(u_{h}^{n}\right)-H_{\delta}\left(v_{h}^{n-1}\right), \theta_{h}\right)
$$

for all $\theta_{h} \in \mathcal{U}_{h}$ which provides

$$
v_{h}^{n}=\tau \Pi r\left(u_{h}^{n}\right)-\Pi\left(v_{h}^{n-1}-\tau H_{\delta}\left(v_{h}^{n-1}\right)\right)
$$

as $v_{h}^{n}$ and $v_{h}^{n-1}$ are in $\mathcal{P}_{1}$. Next, note that $\left(v_{h}^{n-1}-\tau H_{\delta}\left(v_{h}^{n-1}\right)\right)$ is a Lipschitz function of $v_{h}^{n-1}$ with Lipschitz constant $1-\frac{\tau}{\delta}$. Hence, using the above Proposition 4.6, we have

$$
\left\|\nabla \Pi\left(v_{h}^{n-1}-\tau H_{\delta}\left(v_{h}^{n-1}\right)\right)\right\| \leq\left(1-\frac{\tau}{\delta}\right)\left\|\nabla v_{h}^{n-1}\right\| \leq\left\|\nabla v_{h}^{n-1}\right\|
$$

as we choose $\delta=O(\sqrt{\tau})$. This gives,

$$
\left\|\nabla v_{h}^{n}\right\|-\left\|\nabla v_{h}^{n-1}\right\| \leq \tau L_{r}\left\|\nabla u_{h}^{n}\right\|
$$

by first considering pointwise gradients and then taking the $L^{2}(\Omega)$ norm. Summing over $n=1, \ldots, k$ we obtain

$$
\left\|\nabla v_{h}^{k}\right\| \leq\left\|\nabla v_{I}\right\|+\sum_{n=1}^{k} \tau L_{r}\left\|\nabla u_{h}^{n}\right\|
$$

which implies that (since, this holds for any $k \in \mathbb{Z}_{+}, k \leq N$ )

$$
\sup _{k}\left\|\nabla v_{h}^{k}\right\| \leq C .
$$

Using the definition of $V_{h}^{\tau}$, we have

$$
\nabla V_{h}^{\tau}=\nabla u_{h}^{n}+\frac{t-t_{n-1}}{\tau} \nabla u_{h}^{n-1}
$$

and taking $L^{2}$ norm on both sides,

$$
\left\|\nabla V_{h}^{\tau}\right\|^{2} \leq\left\|\nabla v_{h}^{n}\right\|^{2}+\left\|\nabla v_{h}^{n-1}\right\|^{2} .
$$

Integrate in time and use preceding bound (4.26) to conclude

$$
\int_{0}^{T} \int_{\Omega}\left|\nabla V_{h}^{\tau}\right|^{2} d x d t \leq C
$$

The estimate above provides a weak convergence of $V_{h}^{\tau}$ in $L^{2}\left(0, T ; H^{1}(\Omega)\right)$ and combining with $\partial_{t} V_{h}^{\tau} \in$ $L^{2}\left(0, T ; L^{2}(\Omega)\right)$ provides strong convergence of $V_{h}^{\tau}$ in $L^{2}\left(0, T ; L^{2}(\Omega)\right)$. 
4.4 Limit equations In what follows, we show that these limits satisfy the weak formulation (2.7). This is considered in the following theorem.

THEOREM 4.8. The limit triple $(u, v, w)$ satisfy the weak formulation (2.7).

Proof. By the weak convergence, the estimates in Lemma 4.4 carry over for the limit triple $(u, v, w)$. By 4.1, for $\left(U_{h}^{\tau}, V_{h}^{\tau}\right)$ we have

$$
\begin{aligned}
& \int_{0}^{T}\left(\partial_{t} U_{h}^{\tau}, \phi\right) d t+\int_{0}^{T}\left(\nabla U_{h}^{\tau}, \nabla \phi\right) d t+\int_{0}^{T}\left(\mathbf{q}_{h} \cdot \nabla U_{h}^{\tau}, \phi\right) d t+\int_{0}^{T}\left(\partial_{t} V_{h}^{\tau}, \phi\right) d t \\
& =\sum_{n=1}^{N} \int_{t_{n-1}}^{t_{n}}\left(\partial_{t} V_{h}^{\tau}, \phi-\phi_{h}\right)+\sum_{n=1}^{N} \int_{t_{n-1}}^{t_{n}}\left(\partial_{t} U_{h}^{\tau}, \phi-\phi_{h}\right) d t+\sum_{n=1}^{N} \int_{t_{n-1}}^{t_{n}}\left(\nabla U_{h}^{\tau}, \nabla\left(\phi-\phi_{h}\right)\right) d t \\
& +\sum_{n=1}^{N} \int_{t_{n-1}}^{t_{n}}\left(\left(\nabla U_{h}^{\tau}-\nabla u_{h}^{n}\right), \nabla \phi_{h}\right) d t-\sum_{n=1}^{N} \int_{t_{n-1}}^{t_{n}}\left(\mathbf{q}_{h} U_{h}^{\tau},\left(\nabla \phi-\nabla \phi_{h}\right)\right) d t \\
& -\sum_{n=1}^{N} \int_{t_{n-1}}^{t_{n}}\left(\mathbf{q}_{h}\left(U_{h}^{\tau}-u_{h}^{n}\right), \nabla \phi_{h}\right) d t,
\end{aligned}
$$

for all $\phi \in L^{2}\left(0, T ; H_{0}^{1}(\Omega)\right)$, where $\phi_{h}=P_{h} \phi$ is the projection of $\phi$ defined in (2.5). We proceed by showing that the terms on the right are vanishing as the discretization parameters $h$ and $\tau$ are approaching 0 . To this aim we first taken test functions that are $H^{2}$ in space, i.e. $\phi \in L^{2}\left(0, T ; H_{0}^{2}(\Omega)\right)$. As will be seen below, this extra regularity allows us to control terms involving $\nabla\left(\phi-\phi_{h}\right)$. By this we prove that $(u, v)$ satisfy $(2.7)_{1}$, but only for test functions having a better regularity in space. Once this is established, density arguments ensure that the equality is satisfied also for test functions in $L^{2}\left(0, T ; H_{0}^{1}(\Omega)\right)$.

By Lemma 4.5, all terms on the left hand side converge to the desired limits. This is obvious except for the third term where a simple argument takes us through:

$$
\int_{0}^{T}\left(\mathbf{q}_{h} \cdot \nabla U_{h}^{\tau}, \phi\right) d t=\int_{0}^{T}\left(\mathbf{q} \cdot \nabla U_{h}^{\tau}, \phi\right) d t+\int_{0}^{T}\left(\left(\mathbf{q}_{h}-\mathbf{q}\right) \cdot \nabla U_{h}^{\tau}, \phi\right) d t .
$$

The first term on the right hand side of (4.28) passes to the desired limit. We show that the second term vanishes in the limit. Note that $\mathbf{q}_{h}-\mathbf{q} \in L^{\infty}(\Omega)$ and hence, $\left(\mathbf{q}_{h}-\mathbf{q}\right) \nabla U_{h}^{\tau}$ has a weak limit. Now choose $\phi \in L^{2}\left(0, T ; C_{c}^{\infty}(\Omega)\right)$ so that $\phi \in L^{\infty}(\Omega)$ and use the strong convergence of $\mathbf{q}_{h}$ in $L^{2}$ (uniform with respect to $h$ ) to conclude that the weak limit is indeed 0 .

We are thus required to show that the right hand side of (4.27) vanishes in the limit. We treat each term on the right hand side separately. Denote the successive terms in r.h.s. by $\mathcal{I}_{i}$ with $i=1, \ldots, 6$. We have

$$
\left|\mathcal{I}_{1}\right| \leq\left(\int_{0}^{T}\left\|\partial_{t} V_{h}^{n}\right\|^{2} d t\right)^{\frac{1}{2}}\left(\sum_{n=1}^{N} \int_{t_{n-1}}^{t_{n}}\left\|\phi-\phi_{h}\right\|^{2} d t\right)^{\frac{1}{2}} \leq C h\|\nabla \phi\|_{L^{2}\left(0, T ; L^{2}(\Omega)\right)} \searrow 0
$$

as $h \searrow 0$.

For $\mathcal{I}_{2}$ we have the similar argument as in the case of $\mathcal{I}_{1}$. Next,

$$
\left|\mathcal{I}_{3}\right| \leq\left(\int_{0}^{T}\left\|\nabla U_{h}^{\tau}\right\|^{2} d t\right)^{\frac{1}{2}}\left(\sum_{n=1}^{N} \int_{t_{n-1}}^{t_{n}}\left\|\nabla\left(\phi-\phi_{h}\right)\right\|^{2} d t\right)^{\frac{1}{2}} \leq C h\|\phi\|_{L^{2}\left(0, T ; H_{0}^{2}(\Omega)\right)}
$$

and hence, $\mathcal{I}_{3} \searrow 0$ as $h \searrow 0$. Here we use the fact that $\phi \in L^{2}\left(0, T ; H_{0}^{2}(\Omega)\right)$, and hence,

$$
\left\|\nabla\left(\phi-\phi_{h}\right)\right\| \leq C h\|\phi\|_{H_{0}^{2}(\Omega)} .
$$

For $\mathcal{I}_{4}$, we obtain

$$
\left|\mathcal{I}_{4}\right| \leq\left(\sum_{n=1}^{N} \tau\left\|\nabla u_{h}^{n}-\nabla u_{h}^{n-1}\right\|^{2}\right)^{\frac{1}{2}}\left(\sum_{n=1}^{N} \tau\left\|\nabla \phi_{h}\right\|^{2}\right)^{\frac{1}{2}} \rightarrow 0
$$

since $\sum_{n=1}^{N} \tau\left\|\nabla u_{h}^{n}-\nabla u_{h}^{n-1}\right\|^{2} \searrow 0$ because of estimate (4.12).

To continue,

$$
\left|\mathcal{I}_{5}\right| \leq\left(\int_{0}^{T}\left\|\mathbf{q}_{h} U_{h}^{\tau}\right\|^{2} d t\right)^{\frac{1}{2}}\left(\sum_{n=1}^{N} \int_{t_{n-1}}^{t_{n}}\left\|\nabla\left(\phi-\phi_{h}\right)\right\|^{2} d t\right)^{\frac{1}{2}} \leq C h\|\phi\|_{L^{2}\left(0, T ; H_{0}^{2}(\Omega)\right)}
$$


leading to $\mathcal{I}_{5}$ vanishing in the limit. For $\mathcal{I}_{6}$ we have

$$
\left|\mathcal{I}_{6}\right| \leq M_{q}\left(\sum_{n=1}^{N} \tau\left\|u_{h}^{n}-u_{h}^{n-1}\right\|^{2} d t\right)^{\frac{1}{2}}\left(\sum_{n=1}^{N} \tau\left\|\nabla \phi_{h}\right\|^{2} d t\right)^{\frac{1}{2}} \searrow 0
$$

because of (4.11).

Concerning the second equation in (2.7), by $(4.1)_{2}$ we have

$$
\begin{array}{r}
\int_{0}^{T}\left(\partial_{t} V^{\tau}, \theta\right) d t-\int_{0}^{T}\left(r\left(U^{\tau}\right)-W_{h}^{\tau}, \theta\right) d t=\sum_{n=1}^{N} \int_{t_{n-1}}^{t_{n}}\left(\partial_{t} V_{h}^{\tau}, \theta-\theta_{h}\right) d t \\
+\sum_{n=1}^{N} \int_{t_{n-1}}^{t_{n}}\left(r\left(u_{h}^{n}\right)-r\left(U^{\tau}\right), \theta_{h}\right) d t+\sum_{n=1}^{N} \int_{t_{n-1}}^{t_{n}}\left(r\left(U_{h}^{\tau}\right), \theta_{h}-\theta\right) d t \\
+\sum_{n=1}^{N} \int_{t_{n-1}}^{t_{n}}\left(W_{h}^{\tau}-H_{\delta}\left(v_{h}^{n-1}\right), \theta_{h}\right) d t \\
+\sum_{n=1}^{N} \int_{t_{n-1}}^{t_{n}}\left(W_{h}^{\tau}, \theta-\theta_{h}\right) d t .
\end{array}
$$

for all $\theta \in L^{2}\left(0, T ; H_{0}^{1}(\Omega)\right)$, and with $\theta_{h}=P_{h} \theta$. Recall that (see [6])

$$
\left\|\theta-\theta_{h}\right\| \leq C h\|\nabla \theta\| .
$$

We would retrieve the desired limiting equations once we prove that the integrals on the right hand side vanish. Let us denote the successive integrals by $\mathcal{I}_{i}, i=1, \ldots, 5$ respectively. For $\mathcal{I}_{1}$ we get

$$
\left|\mathcal{I}_{1}\right| \leq\left(\int_{0}^{T}\left\|\partial_{t} V_{h}^{\tau}\right\|^{2} d t\right)^{\frac{1}{2}}\left(\sum_{n=1}^{N} \int_{t_{n-1}}^{t_{n}}\left\|\left(\theta-\theta_{h}\right)\right\|^{2} d t\right)^{\frac{1}{2}} \leq C h\|\theta\|_{L^{2}\left(0, T ; H_{0}^{1}(\Omega)\right)}
$$

which vanishes in the limit as $h \searrow 0$. Next, we consider

$$
\left|\mathcal{I}_{2}\right| \leq\left(\sum_{n=1}^{N} \tau L_{r}^{2}\left\|u_{h}^{n}-u_{h}^{n-1}\right\|^{2}\right)^{\frac{1}{2}}\left(\sum_{n=1}^{N} \tau\left\|\theta_{h}\right\|^{2}\right)^{\frac{1}{2}}
$$

and using the estimate (4.11), we obtain $\mathcal{I}_{2} \searrow 0$.

For $\mathcal{I}_{3}$,

$$
\left|\mathcal{I}_{3}\right| \leq L_{r}\left(\int_{0}^{T}\left\|U_{h}^{\tau}\right\|^{2} d t\right)^{\frac{1}{2}}\left(\sum_{n=1}^{N} \int_{t_{n-1}}^{t_{n}}\left\|\left(\theta-\theta_{h}\right)\right\|^{2} d t\right)^{\frac{1}{2}} \leq C h\|\theta\|_{L^{2}\left(0, T ; H_{0}^{1}(\Omega)\right)} \searrow 0
$$

as $h \searrow 0$.

Further, for $\mathcal{I}_{4}$, we use the definition of $W^{\tau}$ and Lipschitz continuity of $H_{\delta}$ to obtain

$$
\left|\mathcal{I}_{4}\right| \leq \sum_{n=1}^{N} \tau \frac{1}{\delta}\left\|v_{h}^{n}-v_{h}^{n-1}\right\|\left\|\theta_{h}\right\| \leq \sum_{n=1}^{N} \tau C \frac{\tau}{\delta}\left\|\theta_{h}\right\|
$$

by using (4.8) and further using $\tau / \delta \searrow 0$ by the construction of $\delta$ we obtain $\mathcal{I}_{4} \searrow 0$. Finally, we treat $\mathcal{I}_{5}$

$$
\left|\mathcal{I}_{5}\right| \leq C\left(\sum_{n=1}^{N} \int_{t_{n-1}}^{t_{n}}\left\|\theta-\theta_{h}\right\|^{2} d t\right)^{\frac{1}{2}} \leq C h\|\nabla \theta\|_{L^{2}\left(0, T ; L^{2}(\Omega)\right)} \searrow 0 \quad \text { as } \quad h \searrow 0
$$

because of (4.29).

Next, we need to prove $w=H(v)$. Since we have $V_{h}^{\tau}$ strongly converging, we also obtain $V_{h}^{\tau} \rightarrow v$ pointwise a.e.. Further,

$$
\left(W_{h}^{\tau}, \theta\right)=(w, \theta)
$$


and by definition, $W_{h}^{\tau}=H_{\delta}\left(V_{h}^{\tau}\right)$; and further,

$$
\left(H_{\delta}\left(V_{h}^{\tau}\right), \theta\right)=(w, \theta) .
$$

As $\tau \searrow 0$, by construction $\delta \searrow 0$. Now, for any given $(t, x) \in \Omega$, either $v>0$ or $v=0$. For the case when $v>0$, we have $W_{h}^{\tau}=1$ and hence $w=1$. For the case when $v=0$, since $\partial_{t} v \in L^{2}(\Omega), \partial_{t} v=0$ leading to $w=r(u)$ with $0 \leq w \leq 1$.

Note that the limit triple $(u, v, w)$ indeed satisfies (2.7), but for test functions having a better regularity in space: $\phi \in L^{2}\left(0, T ; H_{0}^{2}(\Omega)\right)$ and $\theta \in L^{2}\left(0, T ; H_{0}^{1}(\Omega)\right)$. In view of the regularity of $u$ and $v$, density arguments can be employed to show that the limit equations also hold for $\phi \in L^{2}\left(0, T ; H_{0}^{1}(\Omega)\right)$ and $\theta \in L^{2}\left(0, T ; L^{2}(\Omega)\right)$, which completes the proof.

5 Numerical experiments For the numerical experiments, we study different situations. For simplicity and validation exercise, let us consider a 1D situation where we study the concentration profile and the dissolution fronts,

$$
\Omega=(0, L)
$$

with $L=1$ and we choose the following parameters

$$
D=1 e-2, \quad r(u)=k u, \quad k=1, \quad q=1 ;
$$

and the boundary conditions are

$$
u=1, \quad \text { at } \quad x=0, \quad \text { and } \quad \frac{\partial u}{\partial x}=0, \quad \text { at } \quad x=1 .
$$

For initial condition, we make the following choices

$$
u_{I}=1, \quad v_{I}=0.2, \quad x \in[0,1] .
$$

Note that for this initial and boundary conditions, initially, $H\left(v_{I}\right)=1$ as $v_{I}>0$ and

$$
r\left(u_{I}\right)-H\left(v_{I}\right)=u_{I}-H\left(v_{I}\right)=0 .
$$

Hence, in the beginning, it is an equilibrium situation where the dissolution and precipitation processes balance each other. However, this equilibrium is disturbed at $x=0$ because of boundary condition $(u=0)$. This leads to the initiation of dissolution and this process moves forward as $t$ increases. Note also that the dissolution process takes some time before $v$ becomes 0 at $x=0$. We call this time as $t_{s}$. The computation of $t_{s}$ follows from a simple calculation. For $x=0$,

$$
\int_{v_{I}}^{0} \partial_{t} v d t=\int_{0}^{t_{s}} u-H(v) d t=-t_{s}
$$

which gives,

$$
t_{s}=v_{I}=0.2 .
$$

Computations are performed for $t \in(0,1)$ and the discretization in space is obtained on a regular grid of size $h=1 e-3$. Further, we choose standard first order upwinding for the transport term. For the time discretization, we choose $\tau=1 e-3$ and we use regularized Heaviside function (3.1) for the dissolution rate for the 1D problem. For the regularization parameter $\delta$, we choose $\delta=0.1\left(\tau^{0.5}\right)$. 

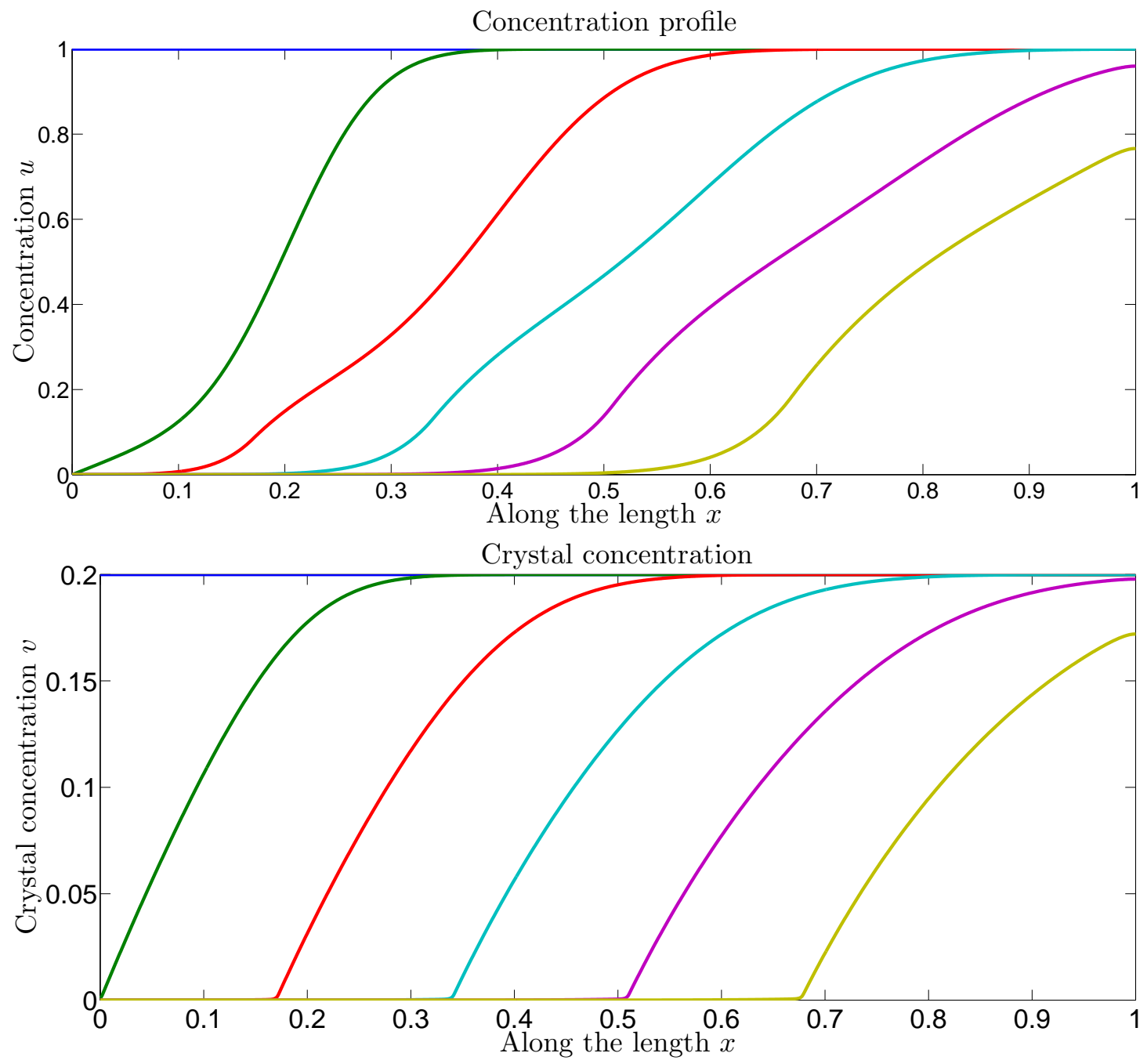

FIG. 5.1. The profiles for the concentration $u$ and the crystal precipitate $v$ for different times, $t=0,0.2,0.4,0.6,0.8,1$. Note that initially $u=1$ and $v=0.2$ and as $t$ increases, the dissolution front moves forward. For the concentration profile, the effect of reactions is clearly visible.

The concentration profiles $u(x, t)$ and the dissolution fronts $v(x, t)$ corresponding to different times have been shown in Figure 5.1. As expected, the dissolution fronts propagate forward almost in a parallel manner and also there is a correspondence with the concentration profile. The 1D case has been studied in literature [14]. There are two quantities that may be of interest here. One is the speed of the dissolution front $Q_{v}$ given by (see Proposition 1.2 of [13])

$$
Q_{v}=q \frac{u^{*}}{u^{*}+v_{I}} \approx 0.833 .
$$

Secondly, the starting time for dissolution process $t_{s}=0.2$ as computed above. These two information are contained in considering the free boundary location $x_{s}$ which is defined as

$$
x_{s}(t):=\left\{\sup _{x \in(0, L)} x, \quad \text { s.t. } \quad v(x, t)=0\right\} .
$$

In Figure 5.2, we plot $x_{s}(t)$ and we notice the time $t_{s}$, the time when the dissolution starts at $x=0$ and the slope of the plot which provides us information about the speed of the free boundary. Numerically, we obtain for the $t_{s}=0.199$ and for the $Q_{v}=0.8464$. We see that the matching is excellent. This provides 


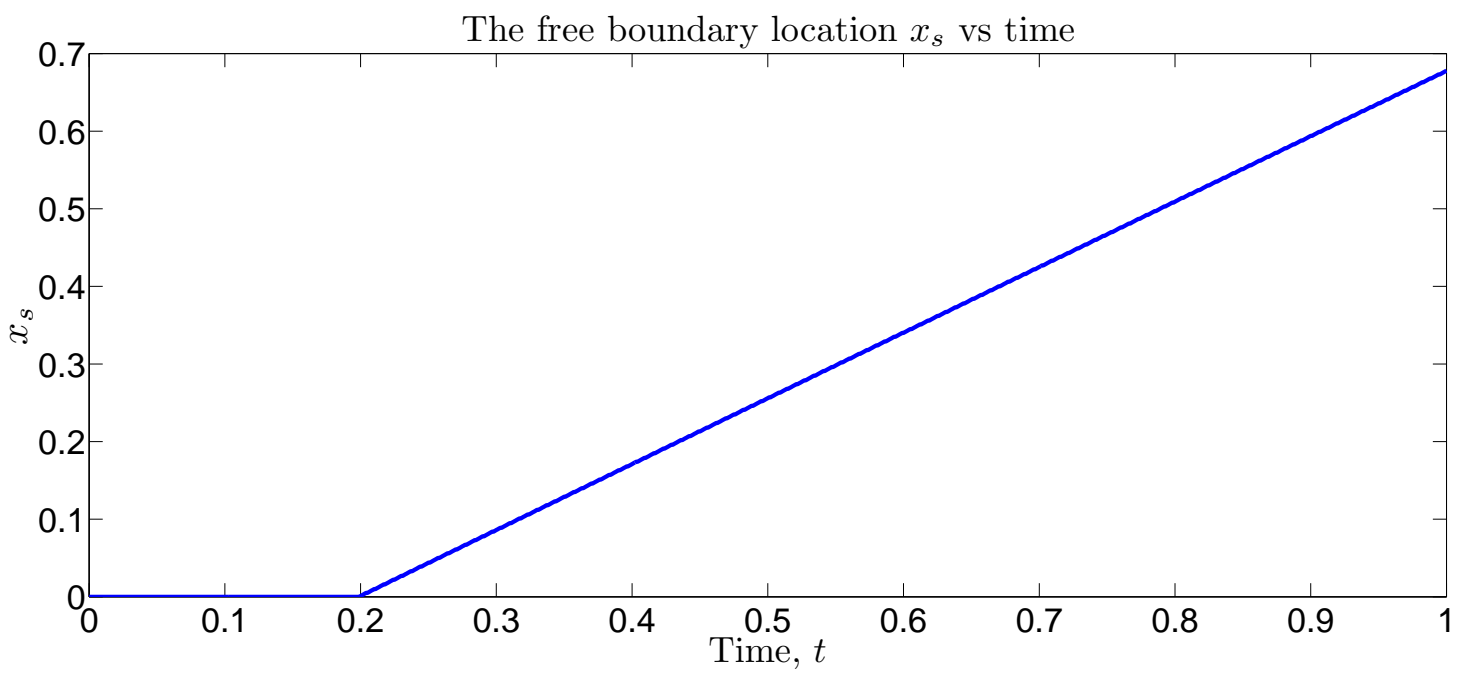

FIG. 5.2. The free boundary location, $x_{s}$. Note the starting time $t_{s}=0.2$ for the beginning of the dissolution front. Also, the slope of the free boundary is equal to $Q_{v}$.

us a validation exercise of the numerical scheme considered here.

For the second situation, we consider a 2D problem. For $\Omega$ we choose

$$
\Omega=(0,1) \times(0,1) .
$$

We choose the following parameters

$$
D=1, \quad r(u)=k u, \quad k=1, \quad \mathbf{q}=0.01 y(1-y) \mathbf{e}_{1} ;
$$

that is, we use a linear reaction rate and for the convection, we have used parabolic profile. With the iterative scheme (4.3) in Section 4.1, the nonlinear reaction rates are also straightforward to compute, however, we stick to linear rates for simplicity of exposition. We choose small convection so that the changes in the concentrations are slow enough for the time scale of our observation $(T=1)$. For choosing the initial conditions, let us define,

$$
\Omega_{v} \subset \Omega ; \quad \Omega_{v}:=\{(x, y) ; 0.4 \leq x \leq 0.6, \quad 0.4 \leq y \leq 0.6\},
$$

that is, there is a small square $\Omega_{v}$ contained in the square domain $\Omega$. Further, we choose, at $t=0, u_{I}=1$ and for $v$

$$
v_{I}=1, \quad(x, y) \in \Omega_{v} ; \quad v_{I}=0 \quad(x, y) \in \Omega \backslash \Omega_{v} .
$$

At $x=0$ we choose $u=1$ and note that for this choice, $r(u)=1$ at $t=0$ and for $\Omega_{v}, H(v)=1$ as $v>0$. Further, for $\Omega \backslash \Omega_{v}$, we put $v=0$ which means that

$$
H(v)=\min (r(u), 1)=1 .
$$

Hence, $\partial_{t} v=u-H(v)=0$ implying that this is an equilibrium situation. For the computations, we choose a uniform space discretization with $h=1 e-2$ and for time stepping $\tau=1 e-3$. For the convection term, we use standard upwinding. For diffusion, convection and reaction, we use implicit in time. We plot the precipitate concentration $v$ in Figure 5.3 for two different times $t=0$ and $t=0.5$. As it is clearly seen, $v$ does not change with time. 

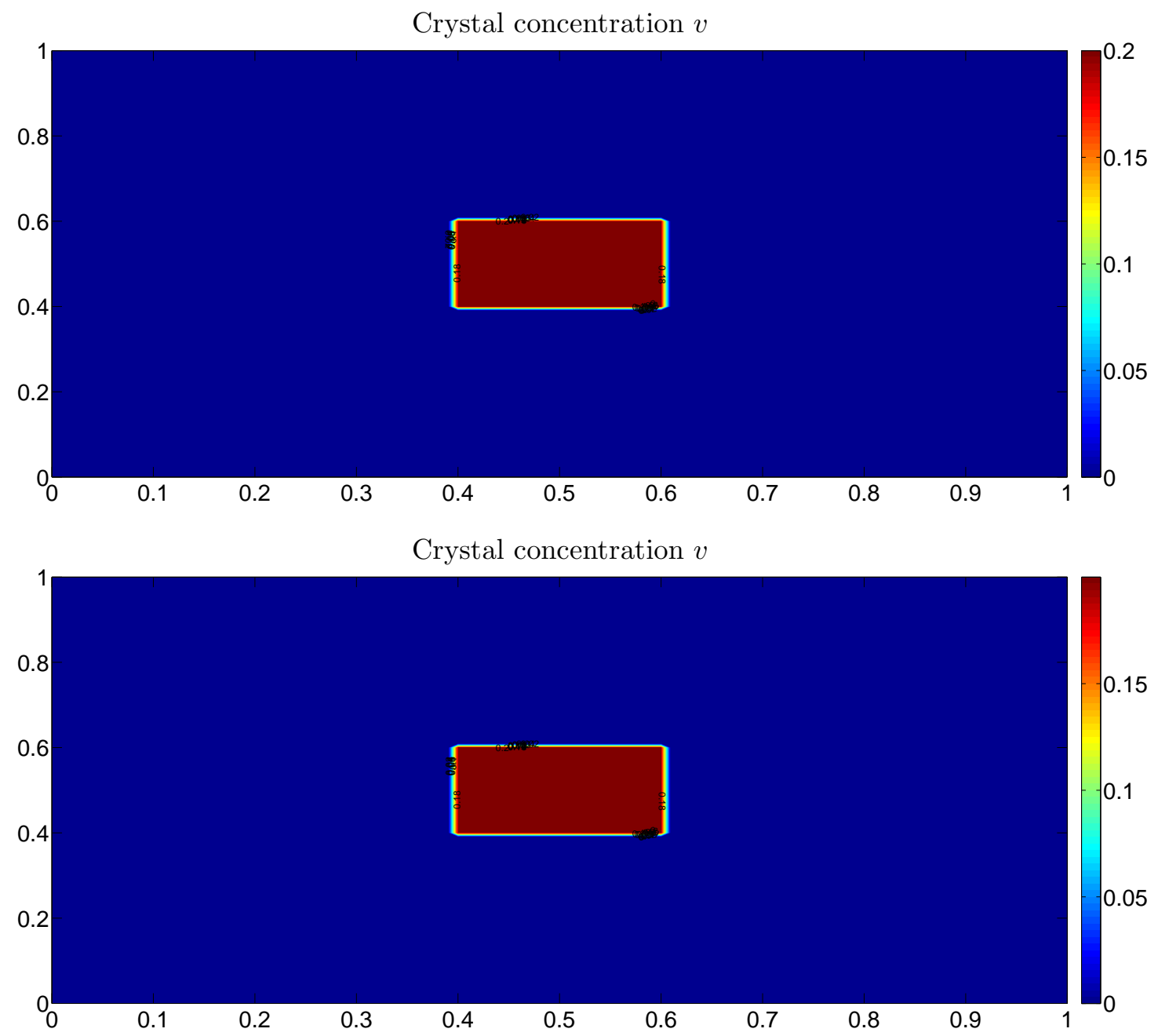

FIG. 5.3. The profiles for the crystal precipitate $v$ for different times, $t=0$ and $t=0.5$. Note that initially $v=0.2$ and as $t$ increases, no changes take place in the profile of $v$. This is an equilibrium situation where the dissolution rates and the precipitation rates annul each other.

Next, we consider the case when we perturb the equilibrium by prescribing the boundary condition $u=0$ at $x=0$. This leads to the beginning of the dissolution process taking place and the concentration of $v$ decreases in $\Omega_{v}$. We have included the concentration profile and the crystal concentration at different times. Notice that the dissolution process proceeds much faster at the left side than on the right side.

6 Conclusions and discussions We have considered both semi-discrete and fully discrete schemes for the macroscale equations. For the fully discrete case, we consider the linear finite elements on the triangular meshes. These schemes have been analyzed for their convergence and the proof relies on a priori estimates and the compactness arguments. To deal with the multi-valued dissolution rate, we consider these numerical schemes along a regularizing sequence. For the a priori estimates, we make sure that the estimates remain independent of the discretization as well as the regularization parameters. The proofs for the semi-discrete and the fully discrete cases follow similar strategy, however, there are some important differences. Whereas the semi-discrete case retains the maximum principle, we have to rely on different estimates in the fully discrete case. Also, the translation estimates to obtain the strong convergence are easily applicable for the semi-discrete situation, the same is not true for the fully discrete case. For the 

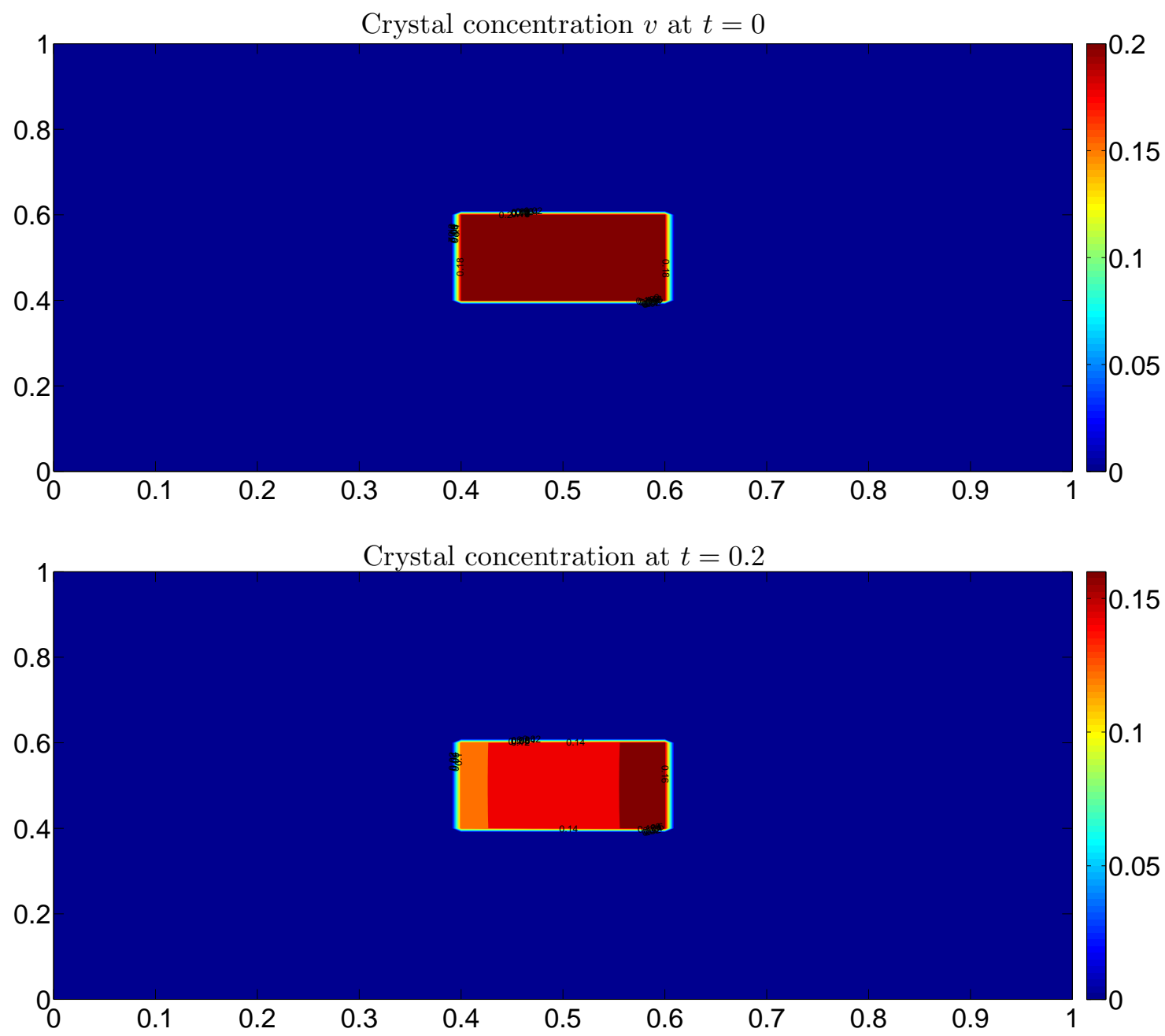

FIG. 5.4. The profiles for the crystal precipitate $v$ for different times, $t=0$ and $t=0.2$. Note that initially $v=0.2$ in $\Omega_{v}$ and as $t$ increases, dissolution process starts taking place because of the boundary condition imposed at $x=0(u=0)$. The net dissolution process then starts taking place and further, we observe that the left hand side experiences more erosion than the right hand side due to the boundary condition effect at $x=0$.

choice of basis functions taken here, that is, piecewise linear elements on a triangle, the usual method of obtaining pointwise estimates does not work in fully discrete case thereby not necessarily having the maximum principle. Next, instead of using the discrete analogue of translation estimates, the properties of Lagrangian interpolation operator on a triangle are used to obtain the strong convergence needed to deal with the nonlinearities.

The numerical experiments have been carried out which present several interesting aspects of the model. In 1D model, we have shown the dissolution fronts propagating forward. Further, we have plotted the free boundary location and shown its evolution. We find an excellent agreement with the theoretical predictions. For the 2D model, we have shown that the model indeed maintains the equilibrium in the absence of crystal $(v=0)$ when the precipitation concentration is not sufficient. Further, for the case when the crystals are present and dissolution process starts taking place due to the boundary effect, we clearly see the evolution of dissolution processes taking place. 


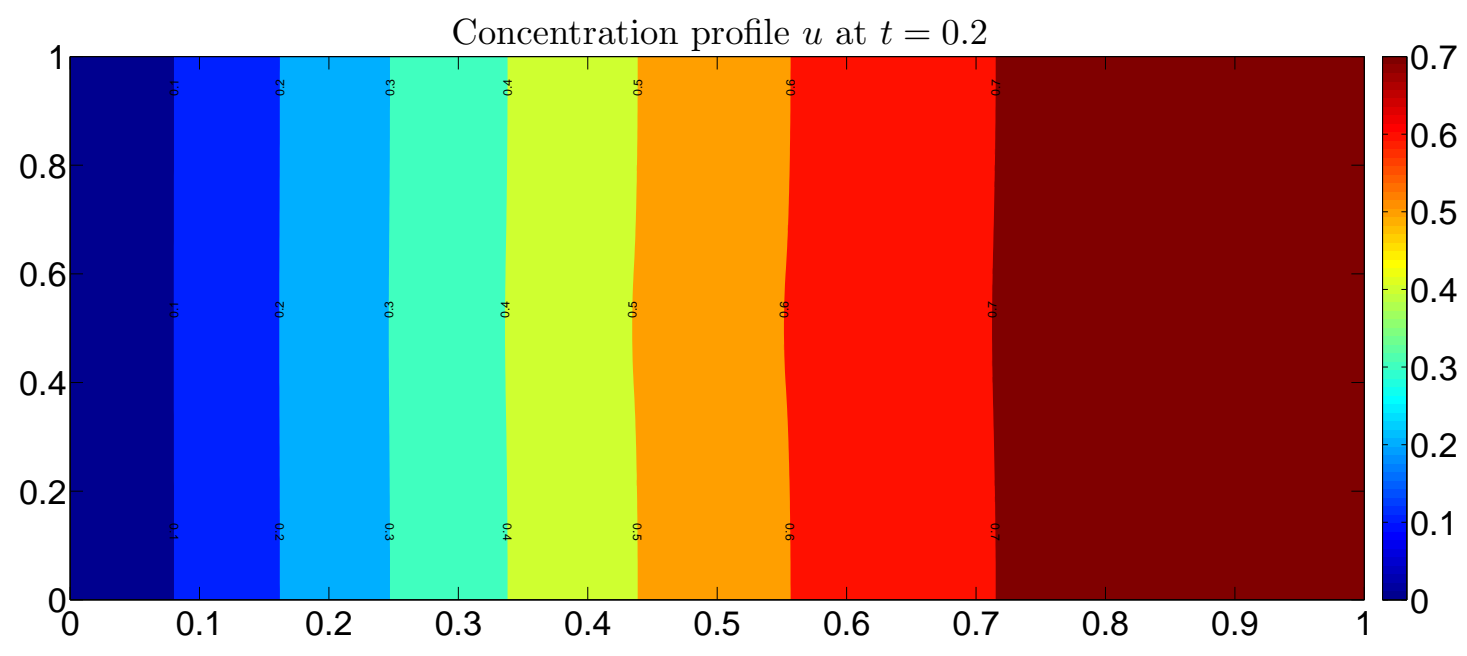

FIG. 5.5. The profiles for the concentration $u$ at $t=0.2$. Note that initially $u=1$ and as $t$ increases, $u$ starts decreasing because of the homogeneous Dirichlet boundary condition at $x=0$. Clearly, $u=v=0$ is the equilibrium situation and we see that the effect of reduction is more prominent on the left side compared to the right side.

Acknowledgement K Kumar would like to thank STW (Project 07796) for the financial support. Part of the work was carried out when K. Kumar and I. S. Pop were visiting Institute of Mathematics, University of Bergen. The support is gratefully acknowledged. 


\section{REFERENCES}

[1] J.W. Barrett and P. Knabner. Finite element approximation of the transport of reactive solutes in porous media. I. Error estimates for nonequilibrium adsorption processes. SIAM J. Numer. Anal., 34(1):201-227, 1997.

[2] J.W. Barrett and P. Knabner. Finite element approximation of the transport of reactive solutes in porous media. II. Error estimates for equilibrium adsorption processes. SIAM J. Numer. Anal., 34(2):455-479, 1997.

[3] N. Bouillard, R. Eymard, R. Herbin, and P. Montarnal. Diffusion with dissolution and precipitation in a porous medium: mathematical analysis and numerical approximation of a simplified model. M2AN Math. Model. Numer. Anal., 41(6):975$1000,2007$.

[4] H. Brezis. Analyse fonctionnelle. Collection Mathématiques Appliquées pour la Maîtrise. [Collection of Applied Mathematics for the Master's Degree]. Masson, Paris, 1983. Théorie et applications. [Theory and applications].

[5] E. Cariaga, F. Concha, I.S. Pop, and M. Sepúlveda. Convergence analysis of a vertex-centered finite volume scheme for a copper heap leaching model. Math. Methods Appl. Sci., 33(9):1059-1077, 2010.

[6] P.G. Ciarlet. The finite element method for elliptic problems. North-Holland Publishing Co., Amsterdam, 1978. Studies in Mathematics and its Applications, Vol. 4.

[7] J. F. Ciavaldini. Analyse numerique d'un problème de Stefan à deux phases par une methode d'éléments finis. SIAM J. Numer. Anal., 12:464-487, 1975.

[8] C. Dawson. Analysis of an upwind-mixed finite element method for nonlinear contaminant transport equations. SIAM J. Numer. Anal., 35(5):1709-1724, 1998.

[9] C. Dawson and V. Aizinger. Upwind-mixed methods for transport equations. Comput. Geosci., 3(2):93-110, 1999.

[10] V. M. Devigne, I. S. Pop, C. J. van Duijn, and T. Clopeau. A numerical scheme for the pore-scale simulation of crystal dissolution and precipitation in porous media. SIAM J. Numer. Anal., 46(2):895-919, 2008.

[11] C. J. van Duijn and P. Knabner. Solute transport through porous media with slow adsorption. In Free boundary problems: theory and applications, Vol. I (Irsee, 1987), volume 185 of Pitman Res. Notes Math. Ser., pages 375-388. Longman Sci. Tech., Harlow, 1990.

[12] C. J. van Duijn and P. Knabner. Solute transport in porous media with equilibrium and nonequilibrium multiple-site adsorption: travelling waves. J. Reine Angew. Math., 415:1-49, 1991.

[13] C. J. van Duijn and P. Knabner. Travelling wave behaviour of crystal dissolution in porous media flow. European J. Appl. Math., 8(1):49-72, 1997.

[14] C. J. van Duijn and I. S. Pop. Crystal dissolution and precipitation in porous media: pore scale analysis. J. Reine Angew. Math., 577:171-211, 2004.

[15] C. M. Elliott. Error analysis of the enthalpy method for the Stefan problem. IMA J. Numer. Anal., 7(1):61-71, 1987.

[16] R. Eymard, D. Hilhorst, and M. Vohralík. A combined finite volume-nonconforming/mixed-hybrid finite element scheme for degenerate parabolic problems. Numer. Math., 105(1):73-131, 2006.

[17] D. Hilhorst and M. Vohralík. A posteriori error estimates for combined finite volume-finite element discretizations of reactive transport equations on nonmatching grids. Comput. Methods Appl. Mech. Engrg., 200(5-8):597-613, 2011.

[18] R. Klöfkorn, D. Kröner, and M. Ohlberger. Local adaptive methods for convection dominated problems. Internat. J. Numer. Methods Fluids, 40(1-2):79-91, 2002. ICFD Conference on Numerical Methods for Fluid Dynamics (Oxford, 2001).

[19] P. Knabner, C. J. van Duijn, and S. Hengst. An analysis of crystal dissolution fronts in flows through porous media. part 1: Compatible boundary conditions. Adv. Water Resour., 18:171-185, 1995.

[20] K. Kumar, T.L. van Noorden, and I. S. Pop. Effective dispersion equations for reactive flows involving free boundaries at the microscale. Multiscale Model. Simul., 9(1):29-58, 2011.

[21] O. A. Ladyzhenskaya and N. N. Ural'tseva. Linear and quasilinear elliptic equations. Translated from the Russian by Scripta Technica, Inc. Translation editor: Leon Ehrenpreis. Academic Press, New York, 1968.

[22] M. Lenzinger and B. Schweizer. Two-phase flow equations with outflow boundary conditions in the hydrophobic-hydrophilic case. Nonlinear Anal., 73(4):840-853, 2010.

[23] I. Metzmacher, F.A. Radu, M. Bause, P. Knabner, and W. Friess. A model describing the effect of enzymatic degradation on drug release from collagen minirods. European Journal of Pharmaceutics and Biopharmaceutics, 67(2):349—360, 2007.

[24] P. Moszkowicz, J. Pousin, and F. Sanchez. Diffusion and dissolution in a reactive porous medium: mathematical modelling and numerical simulations. In Proceedings of the Sixth International Congress on Computational and Applied Mathematics (Leuven, 1994), volume 66, pages 377-389, 1996.

[25] T.L. van Noorden. Crystal precipitation and dissolution in a thin strip. European J. Appl. Math., 20(1):69-91, 2009.

[26] T.L. van Noorden, I. S. Pop, and M. Röger. Crystal dissolution and precipitation in porous media: $L^{1}$-contraction and uniqueness. Discrete Contin. Dyn. Syst., (Dynamical Systems and Differential Equations. Proceedings of the 6th AIMS International Conference, suppl.):1013-1020, 2007.

[27] M. Ohlberger and C. Rohde. Adaptive finite volume approximations for weakly coupled convection dominated parabolic systems. IMA J. Numer. Anal., 22(2):253-280, 2002.

[28] A. Pawell and K.-D. Krannich. Dissolution effects in transport in porous media. SIAM J. Appl. Math., 56(1):89-118, 1996.

[29] I. S. Pop, F. Radu, and P. Knabner. Mixed finite elements for the Richards' equation: linearization procedure. J. Comput. Appl. Math., 168(1-2):365-373, 2004.

[30] J. Pousin. Infinitely fast kinetics for dissolution and diffusion in open reactive systems. Nonlinear Anal., 39(3, Ser. A: Theory Methods):261-279, 2000.

[31] F. A. Radu and I. S. Pop. Newton method for reactive solute transport with equilibrium sorption in porous media. J. Comput. Appl. Math., 234(7):2118-2127, 2010.

[32] F. A. Radu and I.S. Pop. Mixed finite element discretization and newton iteration for a reactive contaminant transport model with nonequilibrium sorption: convergence analysis and error estimates. Comput. Geosci., 15, 2011.

[33] F. A. Radu, I.S. Pop, and S. Attinger. Analysis of an Euler implicit-mixed finite element scheme for reactive solute transport 
in porous media. Numer. Methods Partial Differential Equations, 26(2):320-344, 2010.

[34] B. Rivière and M. F. Wheeler. Non conforming methods for transport with nonlinear reaction. In Fluid flow and transport in porous media: mathematical and numerical treatment (South Hadley, MA, 2001), volume 295 of Contemp. Math., pages 421-432. Amer. Math. Soc., Providence, RI, 2002.

[35] J. Rubin. Transport of reacting solutes in porous media: Relation between mathematical nature of problem formulation and chemical nature of reaction. Water Resources Research, 19:1231-1252, 1983.

[36] R. Temam. Navier-Stokes equations, volume 2 of Studies in Mathematics and its Applications. North-Holland Publishing Co., Amsterdam, third edition, 1984. Theory and numerical analysis, With an appendix by F. Thomasset. 
PREVIOUS PUBLICATIONS IN THIS SERIES:

\begin{tabular}{|c|c|c|c|}
\hline Number & Author(s) & Title & Month \\
\hline I2-O4 & $\begin{array}{l}\text { T.M. van Opstal } \\
\text { E.H. van Brummelen }\end{array}$ & $\begin{array}{l}\text { A Stokes BEM for large- } \\
\text { displacement FSI }\end{array}$ & Febr. 'I2 \\
\hline $12-05$ & $\begin{array}{l}\text { J.H.M. ten Thije } \\
\text { Boonkkamp } \\
\text { J. van Dijk } \\
\text { L. Liu } \\
\text { K.S.C. Peerenboom }\end{array}$ & $\begin{array}{l}\text { Extension of the complete } \\
\text { flux scheme to systems of } \\
\text { conservation laws }\end{array}$ & March 'I2 \\
\hline $12-06$ & $\begin{array}{l}\text { E.N.M. Cirillo } \\
\text { A. Muntean }\end{array}$ & $\begin{array}{l}\text { Dynamics of pedestrians } \\
\text { in regions with no visibility } \\
\text { - a lattice model without } \\
\text { exclusion }\end{array}$ & March 'I2 \\
\hline I2-07 & $\begin{array}{l}\text { J.M.L. Maubach } \\
\text { W.H.A. Schilders }\end{array}$ & $\begin{array}{l}\text { Micro- and macro-block } \\
\text { factorizations for } \\
\text { regularized saddle point } \\
\text { systems }\end{array}$ & Apr. 'I2 \\
\hline I2-O8 & $\begin{array}{l}\text { K. Kumar } \\
\text { I.S. Pop } \\
\text { F.A. Radu }\end{array}$ & $\begin{array}{l}\text { Convergence analysis for a } \\
\text { conformal discretization of } \\
\text { a model for precipitation } \\
\text { and dissolution in porous } \\
\text { media }\end{array}$ & Apr. 'I2 \\
\hline
\end{tabular}

